\title{
Frontostriatal Projections Regulate Innate Avoidance Behavior
}

\author{
Adrienne C. Loewke, ${ }^{1}$ Adelaide R. Minerva, ${ }^{1}$ Alexandra B. Nelson, ${ }^{2,3}$ Anatol C. Kreitzer, $, 2,3,5$ and \\ (iD) Lisa A. Gunaydin ${ }^{1,3,6}$ \\ ${ }^{1}$ Institute for Neurodegenerative Diseases, University of California, San Francisco, San Francisco, California 94158, ${ }^{2}$ Department of Neurology, \\ University of California, San Francisco, San Francisco, California 94158, ${ }^{3}$ Kavli Institute for Fundamental Neuroscience is at University of \\ California, San Francisco, San Francisco, California 94158, ${ }^{4}$ Department of Physiology, University of California, San Francisco, San Francisco, \\ California 94158, ${ }^{5}$ Neurological Disease Institute, Gladstone Institutes, San Francisco, California 94158, and ${ }^{6}$ Department of Psychiatry and \\ Behavioral Sciences, University of California, San Francisco, San Francisco, California 94158
}

The dorsomedial prefrontal cortex (dmPFC) has been linked to avoidance and decision-making under conflict, key neural computations altered in anxiety disorders. However, the heterogeneity of prefrontal projections has obscured identification of specific top-down projections involved. While the dmPFC-amygdala circuit has long been implicated in controlling reflexive fear responses, recent work suggests that dmPFC-dorsomedial striatum (DMS) projections may be more important for regulating avoidance. Using fiber photometry recordings in both male and female mice during the elevated zero maze task, we show heightened neural activity in frontostriatal but not frontoamygdalar projection neurons during exploration of the anxiogenic open arms. Additionally, using optogenetics, we demonstrate that this frontostriatal projection preferentially excites postsynaptic $D_{1}$ receptor-expressing neurons in the DMS and causally controls innate avoidance behavior. These results support a model for prefrontal control of defensive behavior in which the dmPFC-amygdala projection controls reflexive fear behavior and the dmPFC-striatum projection controls anxious avoidance behavior.

Key words: amygdala; anxiety; avoidance; prefrontal cortex; striatum

\section{Significance Statement}

The medial prefrontal cortex has been extensively linked to several behavioral symptom domains related to anxiety disorders, with much of the work centered around reflexive fear responses. Comparatively little is known at the mechanistic level about anxious avoidance behavior, a core feature across anxiety disorders. Recent work has suggested that the striatum may be an important hub for regulating avoidance behaviors. Our work uses optical circuit dissection techniques to identify a specific corticostriatal circuit involved in encoding and controlling avoidance behavior. Identifying neural circuits for avoidance will enable the development of more targeted symptom-specific treatments for anxiety disorders.

\section{Introduction}

Avoiding danger is a fundamental behavior required for survival. However, animals can receive conflicting external cues that indicate both potential risk (inducing avoidance) and potential reward (inducing approach). To resolve this approach-avoidance conflict, the animal must decide how to proceed based on these opposing inputs. One theoretical framework for the resolution of this conflict is reinforcement sensitivity theory, which

\footnotetext{
Received Oct. 5, 2020; revised Apr. 13, 2021; accepted Apr. 16, 2021.

Author contributions: A.C.L., A.C.K., and L.A.G. designed research; A.C.L., A.R.M., A.B.N., and L.A.G. performed research; A.C.L., A.R.M., A.B.N., and L.A.G. analyzed data; A.C.L. and L.A.G. wrote the paper.

This study was funded by a Chan-Zuckerberg Biohub Investigator Award and a Weill Innovation Award to L.A.G. A.C.K. was supported by National Institutes of Health Grant R01-NS-064984.

The authors declare no competing financial interests.

Correspondence should be addressed to Lisa A. Gunaydin at lisa.gunaydin@ucsfedu.

https://doi.org/10.1523/JNEUROSCI.2581-20.2021

Copyright $\odot 2021$ the authors
}

involves the following three opposing systems: the behavioral activation system (BAS), which responds to potential rewards; the fight/flight system (FFS), which responds to imminent threats; and the behavioral inhibition system (BIS), which responds to conflicting drives toward a goal (via BAS) and away from it (via FFS; Corr, 2004; Bijttebier et al., 2009). According to this theory, activation of the BIS leads to a risk-assessment period or a delay in action selection, during which more external information can be received (Corr, 2002; Blanchard et al., 2011). While this response is generally adaptive, it can shift toward a maladaptive overestimation of potential threats in individuals with anxiety disorders (Beck, 1979) - an overactivated BIS leads to excessive risk assessment (e.g., hypervigilance, rumination) and persistent avoidance that can produce severe psychosocial impairment. Compared with our mechanistic understanding of reflexive defensive behaviors such as freezing, little is known about the neural circuit dynamics 
underlying approach-avoidance conflict, representing a major gap in our understanding of anxiety disorders. Identifying the neural circuits underlying avoidance behaviors is critical for developing more targeted symptom-specific treatments.

While reinforcement sensitivity theory offers a conceptual framework for how approach-avoidance conflict may be resolved, it lacks a concrete mapping onto specific brain circuits. The BIS is fundamentally a decision-making system, with inputs from the surrounding environment and outputs that delay action selection. One candidate neural structure for this function is the medial prefrontal cortex ( $\mathrm{mPFC})$, which has been implicated in decision-making (Coutlee and Huettel, 2012; Domenech and Koechlin, 2015), cost-benefit analysis (Shafiei et al., 2012; Hosokawa et al., 2013), and goal-directed actions (Peters et al., 2005; Grace et al., 2007; Pinto and Dan, 2015; Gourley and Taylor, 2016) - all central components of the response to approach-avoidance conflict. Additionally, the mPFC receives contextual and valence information (e.g., from the hippocampus and amygdala; McDonald, 1991; Carr and Sesack, 1996; Hoover and Vertes, 2007) and projects to downstream basal ganglia targets involved in movement and action selection (Groenewegen et al., 1997; Sesack and Grace, 2010), making it well situated to directly control avoidance behaviors based on environmental cues. In rodents, the mPFC is divided into two subregions thought to play opposing roles in defensive behaviors. Dorsomedial PFC (dmPFC), or prelimbic cortex, is implicated in fear expression (Corcoran and Quirk, 2007; Burgos-Robles et al., 2009; Sotres-Bayon et al., 2012), whereas ventromedial PFC, or infralimbic cortex, is implicated in fear extinction (Sotres-Bayon and Quirk, 2010; Sierra-Mercado et al., 2011; Do-Monte et al., 2015). Additionally, altered prefrontal activity has been associated with anxiety disorders (Zhao et al., 2007; Bryant et al., 2008; Qiu et al., 2011), and rodent in vivo electrophysiological recordings have shown that single units within the $\mathrm{MPFC}$ represent aspects of innate avoidance tasks (Adhikari et al., 2011). However, the mPFC is a highly heterogeneous region with many downstream targets, making it difficult to identify which projection-defined mPFC subpopulations are causally involved in innate avoidance behavior. While activity in the $\mathrm{dmPFC}$-amygdala projection has long been associated with fear expression, optogenetic modulation of this projection has no effect on innate avoidance behavior (Adhikari et al., 2015), suggesting the involvement of an alternative dmPFC projection.

One such potential dmPFC target is the striatum, which controls movement and action selection through the following two subpopulations of medium spiny neurons (MSNs): direct-pathway MSNs, expressing $\mathrm{D}_{1}$-type dopamine receptors that promote movement; and indirect-pathway MSNs, expressing $\mathrm{D}_{2}$-type dopamine receptors that inhibit movement. Ventral and dorsomedial aspects of striatum receive prominent innervation from the dmPFC (Sesack et al., 1989; Gabbott et al., 2005) and form basal ganglia circuits that are involved in cognitive/affective behaviors (Alexander et al., 1986; Wiesendanger et al., 2004). Previous studies investigating the role of the striatum in anxiety disorders have primarily focused on the ventral striatum for its role in affective processing (Cardinal et al., 2002; Christakou et al., 2004; Schott et al., 2008), whereas the dorsomedial striatum (DMS) has traditionally been implicated in locomotion (Graybiel et al., 1994). However, the DMS also plays an important role in regulating reinforcement (Kravitz et al., 2012; Kravitz and Kreitzer, 2012), decision-making (Balleine et al., 2007), and several types of avoidance behavior (Green et al., 1967; Rothman and Glick,
1976; Aupperle and Martin, 2010; Aupperle et al., 2015; LeBlanc et al., 2018). Notably, the dmPFC-DMS circuit is involved in decision-making under conflict (Friedman et al., 2015), a key component of the risk-assessment basis of innate avoidance behavior. In a human approach-avoidance conflict task, conflict trials elicited greater caudate (DMS in rodents) activation than non-conflict trials (Aupperle et al., 2015). Recently, DMS D 2 MSNs were shown to control innate avoidance behavior (LeBlanc et al., 2018).

Despite separate lines of evidence that the dmPFC and the DMS are relevant to anxiety and avoidance behavior, no studies have directly examined the role of $\mathrm{dmPFC}$ inputs to the DMS in modulating that behavior. Here, we test the importance of this frontostriatal projection in innate avoidance behavior using a combination of optical circuit-dissection techniques to both record (via fiber photometry) and manipulate (via optogenetics) the neural activity of this projection during the elevated zero maze (EZM) task, which measures innate avoidance of risky anxiogenic environments by quantifying the amount of time animals explore "open arms" (exposed and brightly lit platforms with greater risk of predation) compared with the safer "closed arms" with walls. Additionally, we use slice electrophysiology to address how dmPFC inputs influence the activity of downstream striatal neurons. These studies highlight the importance of dmPFCDMS projection neurons in encoding and controlling anxietyrelated behaviors.

\section{Materials and Methods}

Experimental design and statistical analyses. Wild-type C57BL/6J mice were used for all groups.

Fiber photometry experiments estimated the required sample size ( $n=4$ mice), which was obtained through power analysis calculations (two-sided, $\alpha=0.05$; power $=0.8$; estimated effect size $=3$ ) based on the estimated effect size from preliminary data and previous similar studies (Kim et al., 2017). Sex distribution of animals used for fiber photometry experiments is as follows: $\mathrm{dmPFC}$ cell body (photometry and behavior): six female, five male (GCaMP mice); five female, four male [eYFP (enhanced yellow fluorescent protein) mice]; dmPFC-basolateral amygdala (BLA) projection (photometry): three female, six male (GCaMP mice); five female, seven male (eYFP mice); dmPFC-BLA projection (behavior): four female, seven male (GCaMP mice); five female, seven male (eYFP mice); dmPFC-DMS projection (photometry): three female, seven male (GCaMP mice); four female, six male (eYFP mice); and dmPFC-DMS projection (behavior): five female, eight male (GCaMP mice); four female, six male (eYFP mice).

Optogenetic experiments estimated the required sample size $(n=7$ mice), which was obtained through power analysis calculations (twosided, $\alpha=0.05$; power $=0.8$; estimated effect size $=1.7$ ) based on the estimated effect size from preliminary data and previous similar studies (Tye et al., 2011).

Sex distribution of optogenetics experiments were as follows: dmPFC cell body channelrhodopsin-2 (ChR2): four female, six male (ChR2 mice); three female, six male (eYFP mice); dmPFC cell body halorhodopsin (NpHR): four female, six male (ChR2 mice); three female, seven male (eYFP mice); dmPFC-DMS projection ChR2: nine male (ChR2 mice); eight male (eYFP mice); and dmPFC-DMS projection NpHR: seven female, five male (NpHR mice); seven female, two male (eYFP mice).

Slice electrophysiology experiments estimated required sample size ( $n=5$ pairs) was obtained through power analysis calculations (twosided, $\alpha=0.05$; power $=0.9$; estimated effect size $=1.87$ ) based on the estimated effect size from preliminary data and previous similar studies (Gittis et al., 2010).

Statistical analysis was performed with Prism 7 (GraphPad Software). Normality was tested with D'Agostino-Pearson normality test. For fiber photometry analysis, paired $t$ test (two-tailed, assume 
Gaussian distribution), unpaired $t$ test (two-tailed, assume Gaussian distribution), simple linear regression, and two-way repeated-measures (RM) ANOVA with Sidak's correction for multiple comparisons (assume sphericity) was used. For optogenetics analysis, two-way repeated-measures ANOVA with Sidak's correction for multiple comparisons (assume sphericity) was used. For slice electrophysiology, Wilcoxon signed-rank test was used.

Animal subjects. We used male and female wild-type C57BL/6J mice (The Jackson Laboratory), $\operatorname{Tg}$ (Drd1a-cre)EY217Gsat mice (The Jackson Laboratory), and Drdla-tdTomato mice (Shuen et al., 2008), all on a C57BL/6J background. Animals were raised in normal light conditions (12 h light/dark cycle), and fed and watered ad libitum. All experiments were conducted in accordance with procedures established by the Institutional Animal Care and Use Committee at the University of California, San Francisco.

Stereotaxic surgery, viral injections, and fiber-optic cannula implantation. Surgeries were performed on mice at 10-14 weeks of age. Mice were anesthetized using $5.0 \%$ isoflurane at an oxygen flow rate of $1 \mathrm{~L} /$ min and placed on top of a heating pad in a stereotaxic apparatus (Kopf Instruments). Anesthesia was maintained with $1.5-2.0 \%$ isoflurane for the duration of the surgery. Respiration and toe pinch response were monitored closely. Slow-release buprenorphine $(0.5 \mathrm{mg} / \mathrm{kg})$ and ketoprofen $(1.6 \mathrm{mg} / \mathrm{kg})$ were administered subcutaneously at the start of surgery. The incision area was shaved and cleaned with ethanol and betadine. Lidocaine $(0.5 \%)$ was administered topically on the scalp. An incision was made along the midline, and bregma was measured. Virus was injected (as described below) using a $10 \mu \mathrm{l}$ nanofil syringe (World Precision Instruments) with a 33 gauge beveled needle. We used an injection rate of $100 \mathrm{nl} / \mathrm{min}$ with a $10 \mathrm{~min}$ delay before retracting the needle. Mice recovered in a clean cage on top of a heating pad, and a subsequent injection of ketoprofen $(1.6 \mathrm{mg} / \mathrm{kg})$ was given the following day.

For fiber photometry, we injected $500 \mathrm{nl}$ of AAV5-CaMKIIGCaMP6f or AAV5-CaMKII-eYFP into the dmPFC to record pyramidal neuron activity; to record $\mathrm{dmPFC}-\mathrm{DMS}$ and $\mathrm{dmPFC}-\mathrm{BLA}$ projection neurons, we injected $1500 \mathrm{nl}$ of AAV1-Syn-Flex-GCaMP6m or AAV5EF1a-DIO-eYFP into the dmPFC, and either $350 \mathrm{nl}$ each of CAV2-Cre and hSyn-mCherry in the DMS or $250 \mathrm{nl}$ each in the BLA. Injection coordinates (in millimeters relative to bregma) were as follows: dmPFC: 1.8 anteroposterior (AP), -0.35 mediolateral (ML), -2.6 dorsoventral (DV); DMS: 0.8 AP, $-1.5 \mathrm{ML},-3.5 \mathrm{DV}$; BLA: $-1.4 \mathrm{AP},-3.3 \mathrm{ML},-4.9$ DV. For all fiber photometry experiments, we implanted a $2.5 \mathrm{~mm}$ metal fiber-optic cannula with a $400 \mu \mathrm{m}$ fiber-optic stub (Doric Lenses) in the dmPFC and waited $4-5$ weeks for viral expression. Implant coordinates for the $\mathrm{mPFC}$ were $1.8 \mathrm{AP},-0.35 \mathrm{ML},-2.4 \mathrm{DV}$.

For $\mathrm{dmPFC}$ cell body and projection optogenetic experiments, we injected either $500 \mathrm{nl}$ (cell body) or $800 \mathrm{nl}$ (projection) of 1:3 diluted AAV5-CaMKII-ChR2-eYFP or undiluted AAV5-CaMKII-NpHR3.0eYFP into the dmPFC. For control eYFP mice, we injected undiluted AAV5-CaMKII-eYFP. The NpHR was injected bilaterally for the projection optogenetic experiments. Injection coordinates for the mPFC were $1.8 \mathrm{AP},-0.35 \mathrm{ML},-2.6 \mathrm{DV}$. We implanted a $1.25 \mathrm{~mm}$ ceramic ferrule with $200 \mu \mathrm{m}$ fiber-optic stub (Thorlabs) in either the dmPFC (cell body) or the DMS (projection). Implantation coordinates were as follows: dmPFC: $1.8 \mathrm{AP},-0.3 \mathrm{ML},-2.3 \mathrm{DV}$; DMS: $0.9 \mathrm{AP},-1.0 \mathrm{ML},-3.0 \mathrm{DV}$. For NpHR projection optogenetic surgeries, two fiber-optic cannulas were inserted bilaterally into the DMS.

All viruses were obtained from Addgene, UNC Vector Core, or Institut de Génétique Moléculaire de Montpellier (Montpellier, France).

Elevated zero maze/elevated plus maze. The EZM was custom made using matte white plastic for the floor and closed arm walls, and clear plastic for the inner wall of the closed arms (dimensions: diameter, $55 \mathrm{~cm}$; platform, $30 \mathrm{~cm}$; walls, $60 \mathrm{~cm}$ ). Mice were initially placed in a closed arm. The EZM sessions lasted $15 \mathrm{~min}$ for fiber photometry recording experiments and $25 \mathrm{~min}$ for optogenetic manipulation experiments. Time spent in open arms and closed arms was recorded and quantified by Ethovision XT software (Noldus).

Fiber photometry recording and analysis. In vivo calcium data were acquired using a custom-built rig based on a previously described setup (Lerner et al., 2015). This setup was controlled by an RZ5P fiber photometry processor (TDT) and Synapse software (TDT). The RZ5P/ Synapse software controlled a four-channel LED Driver (model DC4100, Thorlabs), which in turn controlled two fiber-coupled LEDs: $470 \mathrm{~nm}$ for GCaMP stimulation and $405 \mathrm{~nm}$ to control for artifactual fluorescence (catalog \#M470F3, \#M405FP1, Thorlabs). These LEDs were sinusoidally modulated at $210 \mathrm{~Hz}(470 \mathrm{~nm})$ and $320 \mathrm{~Hz}(405 \mathrm{~nm})$ and connected to a Fluorescence Mini Cube with four ports (Doric Lenses), and the combined LEF output was connected through a fiber-optic patch cord (0.48 numerical aperture, $400 \mu \mathrm{m}$; Doric Lenses) to the cannula via a ceramic sleeve (Thorlabs). The emitted light was focused onto a Visible Femtowatt Photoreceiver Module (AC low; model 2151, Newport) and sampled at $60 \mathrm{~Hz}$. Video-tracking software (Ethovision, Noldus) was synchronized to the photometry setup using transistor-transistor logic (TTL) pulses generated every $10 \mathrm{~s}$ following the start of the Noldus trial. Raw photoreceiver data were extracted and analyzed using custom scripts in MATLAB (MathWorks). The two output signal data were demodulated from the raw signal based on the LED modulation frequency. To normalize the data and correct for bleaching, the $405 \mathrm{~nm}$ channel signal was fitted to a polynomial over time and subtracted from the $470 \mathrm{~nm}$ GCaMP signal, yielding the $\Delta F / F$ value.

We analyzed neural activity surrounding transitions with both a $1 \mathrm{~cm}$ distance threshold and a $2 \mathrm{~s}$ time threshold. We generated perievent time histograms ( $40 \mathrm{~s}$ window) by time locking the neural activity [change in fluorescence $(\Delta F / F)$ ] to the transitions, and $z$ scored the $\Delta F / F$ values to the mean and SD from the baseline period $(-20$ to $-10 \mathrm{~s})$ for each transition and averaged across animals. We then quantified the change in calcium signal from the baseline period (pre) to the $10 \mathrm{~s}$ following the transition (post). We created spatial heatmaps by dividing the EZM into sections, calculating the mean signal $(\Delta F / F)$ for each section, and normalizing from 0 to 1 for each animal. For peak amplitude and frequency calculations, we first detected all $\mathrm{Ca}^{2+}$ transient peaks throughout the signal using custom peak detection code using a running average method to calculate the peak-to-trough value. We used a $10 \mathrm{~s}$ trough window (window during convolution for finding running average trough) and a $1 \mathrm{~s}$ temporal window (minimum amount of time between peaks). Once peaks were detected, we then calculated the average frequency and amplitude of these peaks in open versus closed arms. Velocity thresholding was achieved by removing epochs where the velocity of the animal was under $7 \mathrm{~cm} / \mathrm{s}$ for $>10 \mathrm{~s}$. This allowed us to compare neural data from epochs of similar activity level in the open and closed arms.

Optogenetic manipulations. For optogenetic stimulation (both ChR2 and eYFP groups), a $473 \mathrm{~nm}$ laser (Shanghai Laser \& Optics Century Co., Ltd.) was used to stimulate dmPFC cell bodies $(1 \mathrm{~mW}, 10 \mathrm{~Hz}, 5 \mathrm{~ms}$ pulse width) and projection fibers in the DMS $(0.5-1 \mathrm{~mW}, 10 \mathrm{~Hz}, 5 \mathrm{~ms}$ pulse width). For optogenetic inhibition (both NpHR and eYFP groups), green light was generated by a $532 \mathrm{~nm}$ laser (Shanghai Laser \& Optics Century Co., Ltd.) and to inhibit dmPFC cell bodies and projection fibers (bilaterally) in the DMS ( $2-5 \mathrm{~mW}$, constant). dmPFC cell body stimulation and inhibition, as well as $\mathrm{dmPFC}-\mathrm{DMS}$ projection stimulation consisted of a $5 \mathrm{~min}$ baseline laser-off period followed by $102 \mathrm{~min}$ alternating laser on/off epochs. dmPFC-DMS projection inhibition consisted of a $5 \mathrm{~min}$ baseline followed by four $5 \mathrm{~min}$ alternating laser on/off epochs.

Slice electrophysiology. For ex vivo (slice) electrophysiology experiments, we injected adult $\mathrm{D}_{1}$-tmt mice with AAV-CaMKII-ChR2-eYFP (see above) in the mPFC. Four to six weeks after surgery, animals were terminally anesthetized with ketamine/xylazine, and transcardially perfused with ice-cold, carbogenated glycerol-based artificial CSF (aCSF) containing the following (in $\mathrm{mM}$ ): 250 glycerol, $2.5 \mathrm{KCl}, 1.2 \mathrm{NaH}_{2} \mathrm{PO}_{4}$, 10 HEPES, $21 \mathrm{NaHCO}_{3}, 5$ D-glucose, $2 \mathrm{MgCl}_{2}$, and $2 \mathrm{CaCl}_{2}$. The brain was dissected and glued to a chuck, and submerged in ice-cold, carbogenated glycerol-based aCSF. Coronal slices $(300 \mu \mathrm{m})$ containing the striatum were cut using a vibrating microtome (Leica) and immediately transferred to a chamber containing warmed $\left(34^{\circ} \mathrm{C}\right)$ carbogenated aCSF containing the following (in mM): $125 \mathrm{NaCl}, 26 \mathrm{NaHCO}_{3}, 2.5 \mathrm{KCl}, 1.25$ $\mathrm{NaH}_{2} \mathrm{PO}_{4}, 12.5$ D-glucose, $1 \mathrm{MgCl}_{2}$, and $2 \mathrm{CaCl}_{2}$. After incubation for $60 \mathrm{~min}$, slices were stored in carbogenated aCSF at room temperature until used for recordings.

For recordings, slices were transferred to a stage-mounted chamber on a microscope (model BX51, Olympus). Slices were superfused with warmed 
carbogenated aCSF $\left(31-33^{\circ} \mathrm{C}\right)$ throughout. The DMS was identified at low power, and the area of greatest terminal field ChR2-YFP expression was chosen for subsequent whole-cell recordings. In a given field under high power, medium-sized ovoid cell bodies were targeted using differential interference contrast optics. The presence or absence of tdTomato fluorescence was used to determine whether an individual cell body belonged to a direct pathway $\left(D_{1}\right)$ or indirect pathway $\left(D_{2}\right)$ neuron. Since tdTomato-negative neurons could include striatal interneurons, we excluded neurons with physiological features of interneurons (membrane tau decay of $<1 \mathrm{~ms}$ ). $\mathrm{D}_{1}$ and $\mathrm{D}_{2}$ neurons were patched in nearby serial pairs, in randomized order. All whole-cell recordings were acquired (filtered at $5 \mathrm{kHz}$ ) using an amplifier (model Multiclamp 700B, Molecular Devices) and digitized $(10 \mathrm{kHz})$ using an analog-to-digital board (model ITC-18, HEKA). Igor Pro 6.0 software and custom acquisition routines (mafPC; courtesy of Matthew A. XuFriedman (Department of Biological Sciences, University at Buffalo, State University of New York, Buffalo, New York, 14260)) were used to acquire and analyze the data.

Neurons were patched in the whole-cell voltage-clamp configuration using borosilicate glass electrodes (3-5 M $\Omega$ ). To record EPSCs, we used a cesium methanesulfonate-based, low chloride internal solution containing the following (in $\mathrm{mm}$ ): $120 \mathrm{CsMeSO}_{3}, 15 \mathrm{CsCl}, 8 \mathrm{NaCl}, 0.5$ EGTA, and 10 HEPES, pH 7.3. The internal chloride concentration was calibrated such that the reversal potential of $\mathrm{GABA}_{\mathrm{A}}$-mediated (disynaptic) IPSCs was $-70 \mathrm{mV}$ (thus currents recorded at $-70 \mathrm{mV}$ were predominantly glutamatergic in origin). Experiments were performed in picrotoxin to pharmacologically isolate EPSCs. mPFC-derived EPSCs were measured at $-70 \mathrm{mV}$ holding potential, evoked using brief ( $3 \mathrm{~ms}$ ) full-field blue $(473 \mathrm{~nm})$ light pulses delivered by a TTL-controlled LED (Olympus) through a ChR2 filter. Light power $(473 \mathrm{~nm})$ was set at 1 $\mathrm{mW}$ at the objective using a light meter (Thorlabs). EPSC amplitude was defined as the average difference between the baseline holding current (0-100 ms before the light pulse) and the peak of the evoked EPSC, averaged over at least five trials (intertrial interval, $20 \mathrm{~s}$ ).

Histology. Following the conclusion of behavioral experiments, animals were anesthetized using $5 \%$ isoflurane and given a lethal dose (1.0 $\mathrm{ml})$ of a cocktail of ketamine/xylazine $(10 \mathrm{mg} / \mathrm{ml}$ ketamine, $1 \mathrm{mg} / \mathrm{ml}$ xylazine). They were then transcardially perfused with $10 \mathrm{ml}$ of $1 \times$ PBS followed by $10 \mathrm{ml}$ of $4 \%$ paraformaldehyde (PFA). Brains were extracted and left in 4\% PFA overnight and then transferred to a $30 \%$ sucrose solution until slicing. The brains were frozen and sliced on a sliding microtome (Leica Biosystems) and placed in cryoprotectant in a well plate. Slices were then washed in $1 \times$ PBS, mounted on slides (Fisher Superfrost Plus, Thermo Fisher Scientific) and air dried (covered). Invitrogen ProLong Gold antifade reagent (Thermo Fisher Scientific) was injected on top of the slices, a coverslip (Slip-rite, ThermoFisher) was placed on top, and the slides were left to dry overnight (covered). Viral injection, fiber photometry cannula implant, and optogenetic cannula implant placements were histologically verified on a fluorescence microscope (Leitz DMRB, Leica).

Confocal imaging and cell counting. A random subset of DIO-eYFP/ CAV2-Cre-injected mice from our experiments was chosen, with all mice having received the same lot number of virus. Mounted slices were imaged on a confocal microscope (Leica SP8). The same gain and laser power were used across each channel, and a $512 \times 512$ image $z$-stack was obtained. Using ImageJ, the maximum projection was created and a $700 \times 700$ pixel box was centered just below the tip of the fiber in which labeled cells were counted. The image was then converted to 16 bit and run through a particle analysis-nucleus counter using the Otsu's thresholding method and a watershed filter to obtain cell counts for each slice.

\section{Data availability}

All data and code are freely available by contacting the corresponding author directly.

\section{Results}

dmPFC pyramidal neurons exhibit task-related neural activity in the EZM

We first characterized the neural activity of undefined dmPFC pyramidal neurons (henceforth referred to as "whole-population
dmPFC") during avoidance behavior. We virally expressed either CaMKII-GCaMP6f or CaMKII-eYFP and implanted an optical fiber $(400 \mu \mathrm{m})$ in the dmPFC to record bulk $\mathrm{Ca}^{2+}$ fluorescence changes during exploration of the EZM (Fig. 1A). To visualize neural activity spatially, we subdivided the maze into sections and calculated the mean $\mathrm{Ca}^{2+}$ signal in each section. We used four sections for each half of the open and closed arms; section 1 was closest to the open/closed transition point, while section 4 was in the middle of the arm (Fig. $1 B$ ). The $\mathrm{Ca}^{2+}$ signal from dmPFC pyramidal neurons was lowest when mice were in the middle of a closed arm (C4), and it increased as mice approached an open arm, with the highest signal occurring in the middle of the open arm (O4; Fig. 1B).

We also examined temporal changes in the neural signal surrounding the open/closed arm transitions. We plotted a perievent time histogram $(\mathrm{PETH})$ of the $\mathrm{Ca}^{2+}$ signal for the $\pm 20 \mathrm{~s}$ surrounding each transition (closed-to-open and open-toclosed). The average $\mathrm{Ca}^{2+}$ signal was generated for the following three different time windows: baseline $(-20$ to $-10 \mathrm{~s})$, pretransition $(-10$ to $0 \mathrm{~s})$, and post-transition $(0-10 \mathrm{~s})$. dmPFC neurons showed a significant increase in signal as mice transitioned from closed to open arms [Fig. 1C,D; two-way RM ANOVA interaction, $F_{(1,329)}=17.7, p<0.0001$; Sidak's multiple comparisons, $p<0.0001$ (GCaMP, pretransition vs post-transition); $p=0.9727$ (eYFP, pretransition vs post-transition); $N=204$ GCaMP transitions, $N=127$ eYFP transitions; $N=11$ GCaMP mice, $N=9$ eYFP mice]. Paralleling the spatial heatmap findings, the increase in $\mathrm{Ca}^{2+}$ signal slightly preceded the transition into the open arms. Conversely, dmPFC neurons showed a significant decrease in signal as animals transitioned from open to closed arms [Fig. 1E,F; two-way RM ANOVA interaction, $F_{(1,374)}=44.25, p<0.0001$; Sidak's multiple comparisons, $p<0.0001$ (GCaMP, pretransition vs post-transition); $p=$ 0.3962 (eYFP, pretransition vs post-transition); $N=226$ GCaMP transitions, $N=150$ eYFP transitions]. Unlike the gradual change in signal seen in the closed-to-open transition, the signal decayed rapidly on return to the closed arms. eYFP animals showed no signal modulation during either transition. We plotted the probability of the mice being in the open arms at any given time point (Fig. 1C,E, inset); the decay slope in the $\mathrm{Ca}^{2+}$ signal tightly parallels the probability that the mouse is in the open arms, and the decay duration matches the average time spent in the open arms. Together, these spatiotemporal changes indicate that, on average, $\mathrm{dmPFC}$ activity increases as the mice approach and enter an open arm and then decreases as they transition back into a closed arm.

To ensure that these neural representations would hold across different maze configurations, we additionally recorded from dmPFC neurons during exploration of the elevated plus maze (EPM), a similar innate avoidance assay. We found that dmPFC neurons show the same modulation of signal during center to open transitions on the EPM as during closed to open transitions on the EZM [Fig. 1G,H; two-way RM ANOVA interaction, $F_{(1,214)}=8.362, p=0.0042$; Sidak's multiple comparisons, $p<$ 0.0001 (GCaMP, pretransition vs post-transition); $p=0.9805$ (eYFP, pretransition vs post-transition); $N=138$ GCaMP transitions, $N=78$ eYFP transitions].

In addition to quantifying changes in neural activity surrounding the transition zone, we compared additional measures of neural activity between the open and closed arms. To visualize the frequency of $\mathrm{Ca}^{2+}$ events, we calculated frequency of event peaks in $5 \mathrm{~s}$ bins and plotted frequency as a function of spatial location in the EZM (Fig. 2A). From a neuronal population 
A

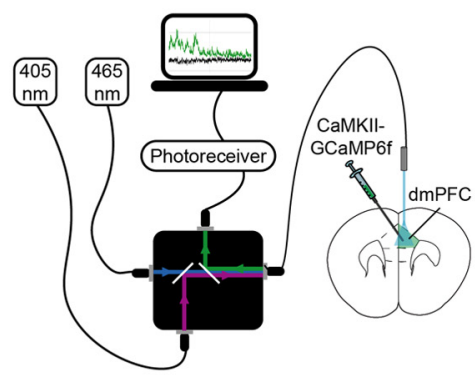

C

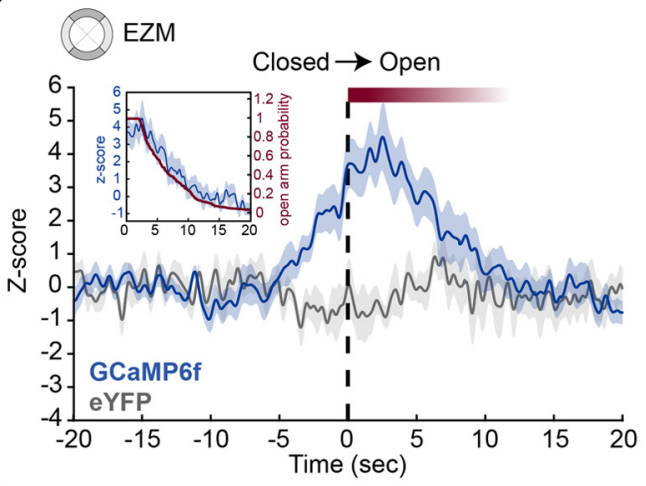

E

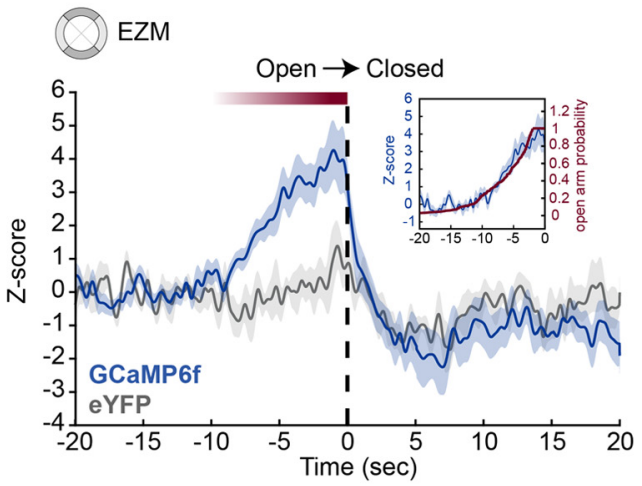

G

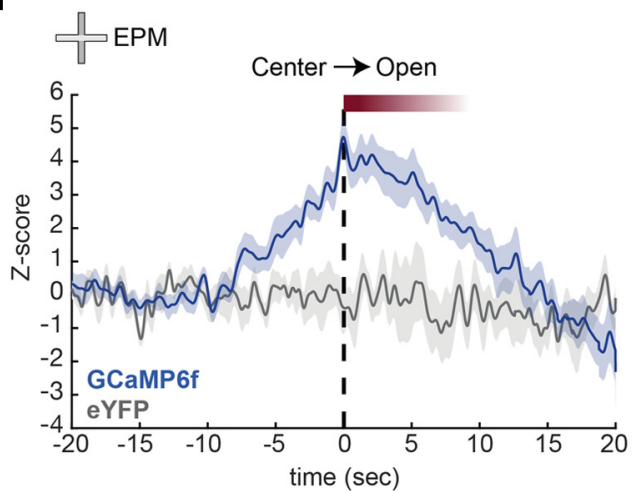

B
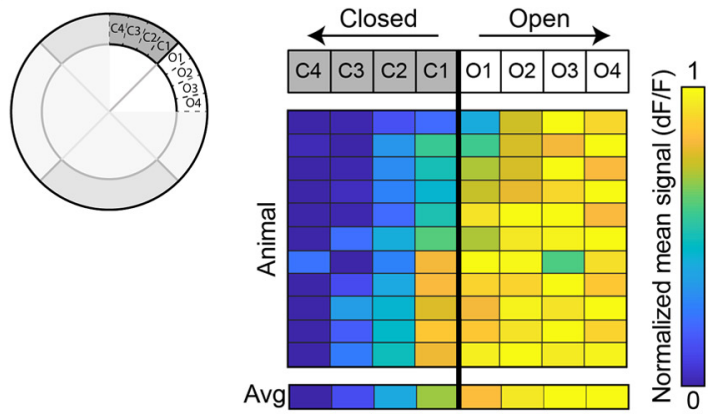

D

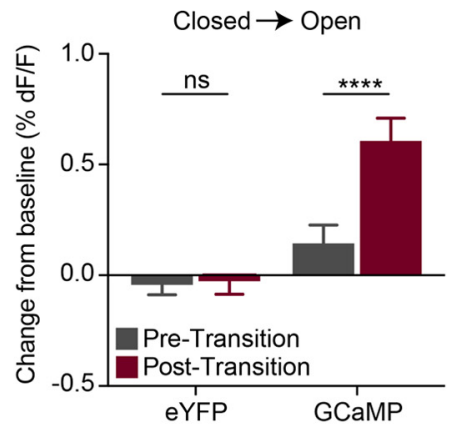

F

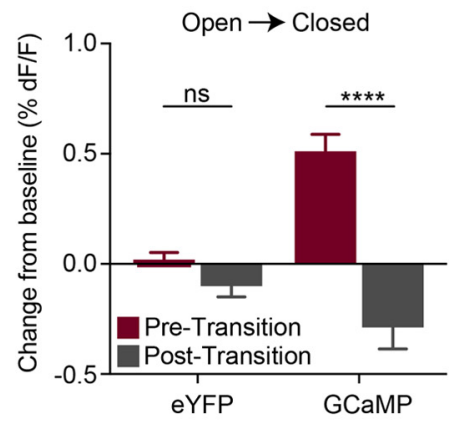

H

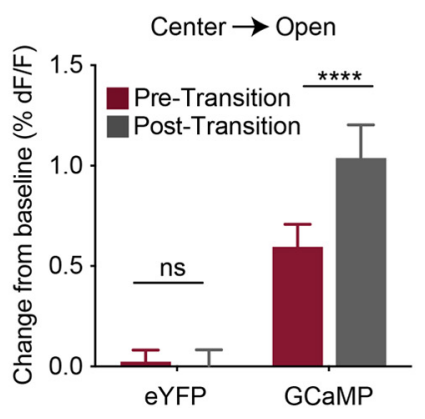

Figure 1. $\mathrm{dmPFC}$ pyramidal neurons exhibit transition-related neural activity in the elevated zero maze. $\boldsymbol{A}$, Fiber photometry recording of dmPFC excitatory neurons expressing GCaMP6f. $\mathrm{Ca}^{2+}$ signals were recorded during exploration of the EZM. B, Left, Schematic of EZM with spatial sectioning. Right, Spatial increase in $\mathrm{Ca}^{2+}$ signal when mice are in the open arms (black line, transition point). C, Perievent time histogram showing temporal increase in $\mathrm{Ca}^{2+}$ signal on transition from closed to open arms (transition at time $=0$; dotted black line). The $\mathrm{Ca}^{2+}$ signal tightly follows the probability that mice are in the open arms (inset, red line). Blue line, Mean \pm standard error of the mean (SEM) for GCaMPGf; gray line, mean \pm SEM for eYFP. D, Ca ${ }^{2+}$ signal (\% dF/F normalized to baseline $\mathrm{dF} / F$; baseline is -20 to $-10 \mathrm{~s})$ is significantly higher in the open arm post-transition (0-10 s) than in the closed arm pretransition $(-10$ to $0 \mathrm{~s})$. $\boldsymbol{E}$, Same as $\mathbf{C}$ for the open to closed arm transition. Mice show a decrease in signal following transition into the closed arms (GCaMP6f: $N=226$ transitions, $N=11$ mice; eYFP: $N=150$ transitions, $N=9$ mice). $F$, Ca ${ }^{2+}$ signal is significantly lower in the closed arm post-transition $(0-10 \mathrm{~s})$ than in the open arm pretransition $(-10$ to $0 \mathrm{~s})$. $G$, Perievent time histogram shows increased $\mathrm{Ca}^{2+}$ signal on transition from the center to open arms (transition at time $=0$; dotted black line). Signal is plotted as $z$ score, normalized using the mean and SD of the baseline period $(-20$ to $-10 \mathrm{~s})$. Blue line, Mean \pm SEM GcaMP; gray line, mean \pm SEM eYFP control signal; gray shading, SEM eYFP control signal). $\boldsymbol{H}, \mathrm{Ca}^{2+}$ signal is significantly higher in the open arm post-transition period (0-10 s) than in the closed arm pretransition period $\left(-10\right.$ to 0 s). No significant difference is seen in the eYFP control signal. Error bars on all bar graphs represent SEM. ${ }^{* * *} p<0.0001$, ns, not significant. 
A

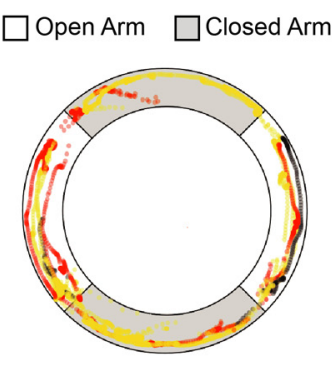

B

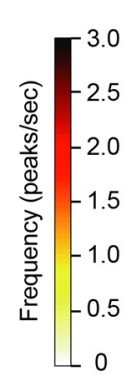

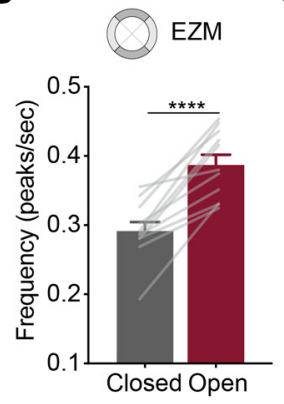

C

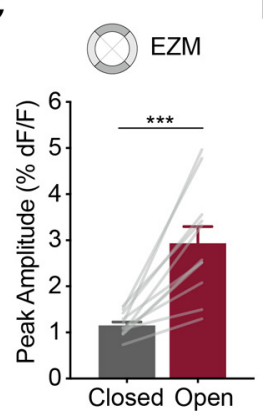

D

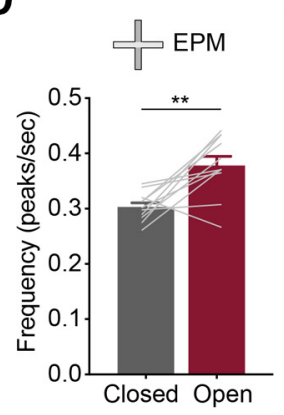

$\mathbf{E}$

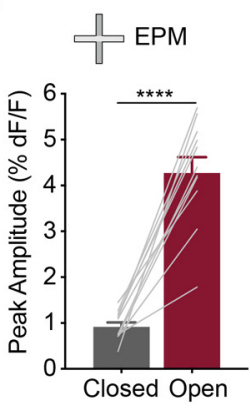

$\mathbf{F}$

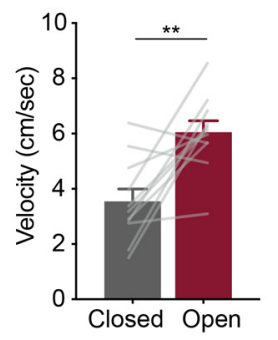

G

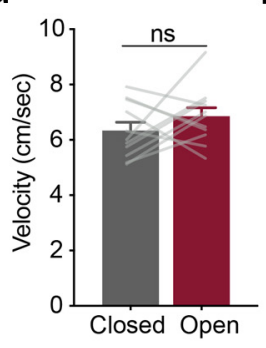

H

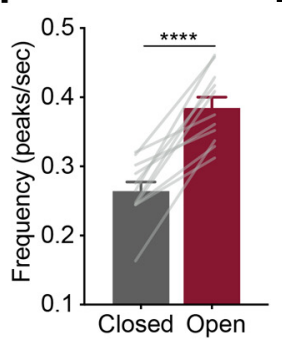

I

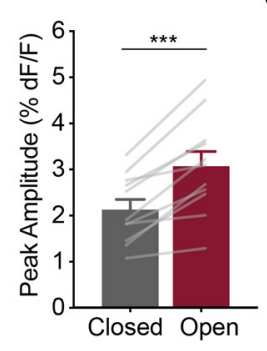

$\mathbf{J}$

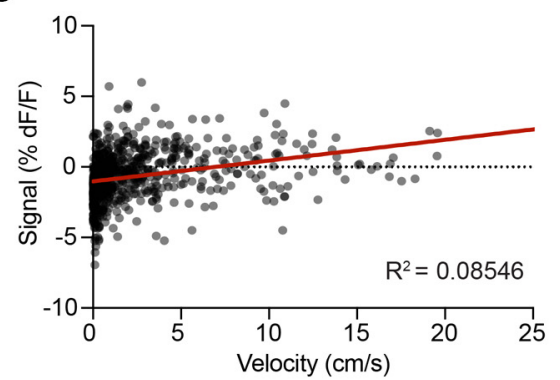

Figure 2. $\mathrm{dmPFC}$ pyramidal neurons exhibit differential encoding of the closed and open arms in the elevated zero maze and elevated plus maze. $\boldsymbol{A}$, Aerial view of the EZM with a representative trajectory of the animal (in $5 \mathrm{~s}$ bins), color coded by the frequency of $\mathrm{Ca}^{2+}$ transients, showing a higher frequency in the open arms than in the closed arms. $\boldsymbol{B}$, Frequency of $\mathrm{Ca}^{2+}$ transients is significantly higher in the open arms than in the closed arms. C, Peak amplitude of $\mathrm{Ca}^{2+}$ transients is significantly higher in the open arms than in the closed arms. D, E, Same as $\boldsymbol{B}$ and $\mathbf{C}$ for EPM data. $\boldsymbol{F}$, Whole-population dmPFC mice show higher velocity of movement in the open arms (maroon) than in the closed arms (gray). $\mathbf{G}$, Once data are velocity corrected (see Materials and Methods), there is no longer a difference. $\boldsymbol{H}$, Frequency of calcium transients is significantly higher in the open arms than in the closed arms (velocity corrected). $\boldsymbol{I}$, Peak amplitude of calcium transients is significantly higher in the open arms than in the closed arms (velocity corrected). J, dmPFC whole-population neurons no correlation between velocity and GCaMP signal. Error bars on all bar graphs represent SEM. ${ }^{* *} p<0.002,{ }^{* * *} p<0.001,{ }^{* * * *} p<0.0001$, ns, not significant.

standpoint, "peaks" in calcium fluorescence could indicate greater synchronous neuronal firing or simply a greater number of active neurons, resulting in bursts of summed activity. dmPFC pyramidal neurons showed a higher frequency of $\mathrm{Ca}^{2+}$ events in the open arms than in the closed arms (Fig. 2B; paired $t$ test, $t=7.121, \mathrm{df}=10, p<0.0001 ; N=11$ mice). dmPFC pyramidal neurons also showed significantly greater average peak amplitude of $\mathrm{Ca}^{2+}$ events in the open arms than in the closed arms (Fig. 2C; paired $t$ test, $t=5.656, \mathrm{df}=10$, $p=0.0002 ; N=11$ mice). $\mathrm{Ca}^{2+}$ events in the open arm of the EPM showed the same increase in frequency (Fig. 2D; paired $t$ test, $t=3.782, \mathrm{df}=10, p=0.0036, N=11$ mice) and peak amplitude (Fig. 2E; paired $t$ test, $t=9.870, \mathrm{df}=10, p<0.0001$; $N=11$ mice) compared with the closed arm.

To control for any differences in velocity of movement in the open arm versus closed arm, we analyzed the neural signal during bouts of similar velocity in the closed and open arms (any bout during which the animal moved $<7 \mathrm{~cm} / \mathrm{s}$ for $\geq 10 \mathrm{~s}$ was discarded). Originally, the bouts in the open arm had higher velocity than bouts in the closed arm (Fig. $2 F$; paired $t$ test, $t=3.858$, $\mathrm{df}=10, p=0.0032 ; N=11$ mice). Our velocity thresholding was successful in selecting only bouts that had similar velocity in open and closed arm (Fig. $2 G$; $t$ test, $t=1.097, \mathrm{df}=10, p=0.2982$; $N=11$ mice). Using this velocity thresholding, we found that these open arm-related changes in neural activity did not depend on velocity (Fig. $2 H, I$; frequency: paired $t$ test, $t=7.196, \mathrm{df}=10$, $p<0.0001$; amplitude: paired $t$ test, $t=6.011, \mathrm{df}=10, p=0.0001$; $N=11$ mice). Additionally, we binned velocity and GCaMP signal from the closed arm (to control for open arm exposure) in 10 $\mathrm{s}$ bins and found no correlation between these variables (Fig. 2J; linear regression, signal $=0.1474 *$ (velocity) $-1.024, R^{2}=$ 0.08546). Together, these results indicate that the activity of
dmPFC pyramidal neurons is lowest in the closed arms, increases as mice approach the open arms, and peaks in the open arms, suggesting that these neurons are encoding aspects of innate avoidance across tasks.

Frontostriatal, but not frontoamygdalar, projection neurons recapitulate whole-population dmPFC activity in the EZM Whole-population recording does not provide projection-specific information about dmPFC neurons involved in innate avoidance behavior and may mask the activity of less represented subpopulations in the dmPFC. We therefore next recorded the activity of projection-defined subpopulations of dmPFC neurons during exploration of the EZM. While the dmPFC-BLA projection has been well studied in fear expression, a recent study showed that this projection is not causally involved in innate avoidance behavior (Adhikari et al., 2015). We thus hypothesized that a different subpopulation of dmPFC neurons-the frontostriatal projection to the DMS-drives the encoding of innate avoidance behavior we observed at the whole-population level. Recently, the DMS was found to have a causal role in innate avoidance behavior in the EZM (LeBlanc et al., 2018), and optogenetic manipulation of the dmPFC-DMS projection causally modulates decision-making under conflict, a prefrontal function relevant to avoidance behavior (Friedman et al., 2015).

To examine the roles of the frontostriatal and frontoamygdalar projections, we used a retrograde viral targeting strategy to express GCaMP6f selectively in cells projecting to either the BLA or DMS (Fig. $3 A, L$ ). We injected a retrograde canine adenovirus, CAV2, carrying Cre recombinase (CAV2-Cre) in the downstream area to allow for the expression of Cre in any neurons projecting to that area. Additionally, we injected a Cre-dependent GCaMP6f in the upstream dmPFC, which allowed for 
A
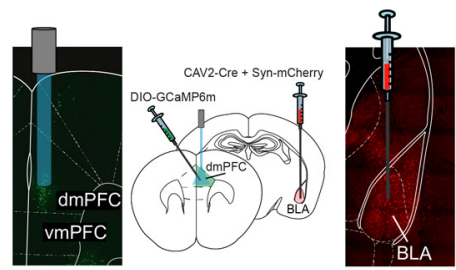

B

E

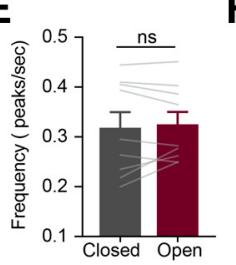

F

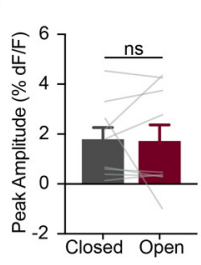

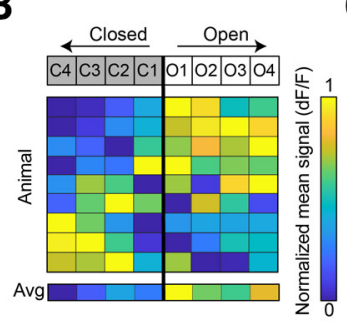

C

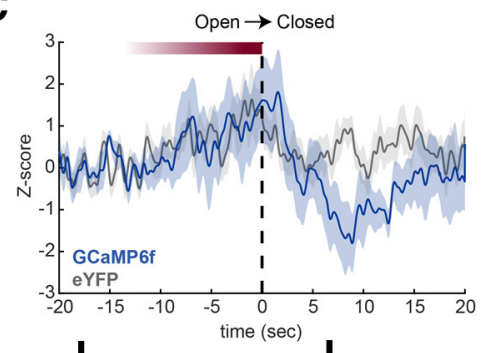

H

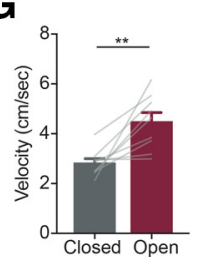

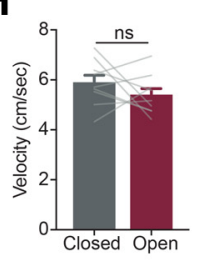

I

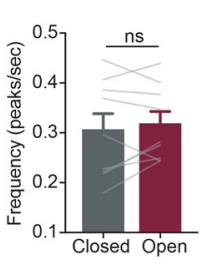

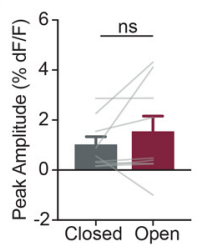

D

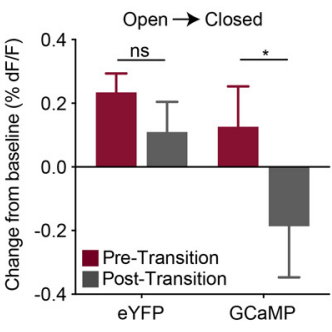

K

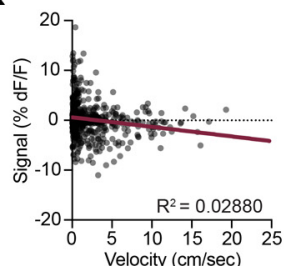

L

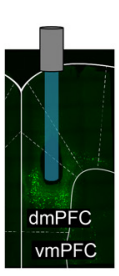

P

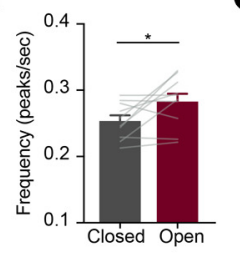

Q

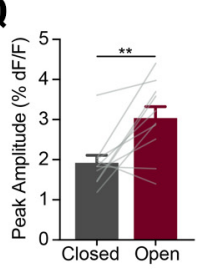

M

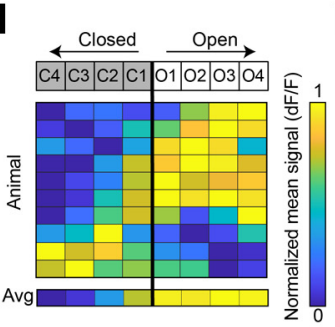

R

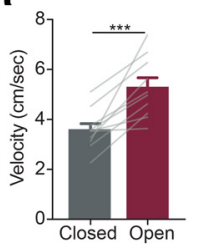

N

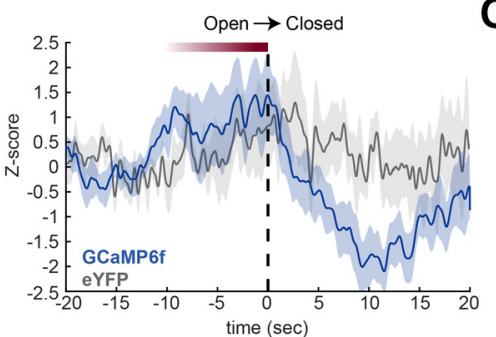

T

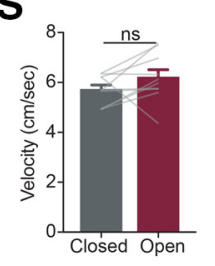

0

U

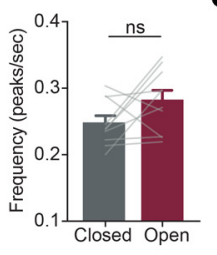

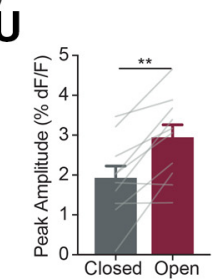

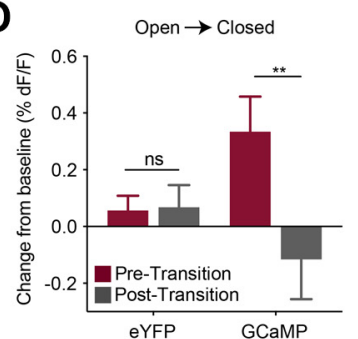

V

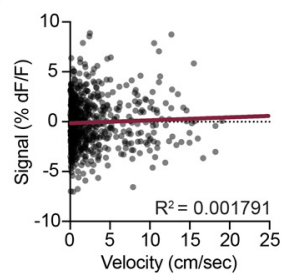

Figure 3. Frontostriatal, but not frontoamygdalar, projection neuron activity recapitulates whole-population dmPFC activity in the elevated zero maze. $\boldsymbol{A}$, Injection schematic with representative histology images showing the targeting of DIO-GCaMP6m to the dmPFC and CAV-Cre/Syn-mCherry to the BLA. B, Spatial analysis of frontoamygdalar projection activity in the EZM showing heterogeneous $\mathrm{Ca}^{2+}$ signal values in the open and closed arms (black line, transition point). Individual animal data are sorted by the average signal across the closed arms (low to high). Average heatmap is plotted below ( $N=9$ mice). C, Perievent time histogram showing temporal decrease in $\mathrm{Ca}^{2+}$ signal on transition from open to closed arms (transition at time $=0$; dotted black line). $D, \mathrm{Ca}^{2+}$ signal (percentage of $\mathrm{dF} / \mathrm{F}$ normalized to baseline $\mathrm{dF} / \mathrm{F}$; baseline, -20 to $-10 \mathrm{~s}$ ) is significantly lower in the closed arm post-transition (0-10 s) than in the open arm pretransition ( -10 to $0 \mathrm{~s}$ ). $\boldsymbol{E}$, No difference in the frequency of $\mathrm{Ca}^{2+}$ transients in the open and closed arms. $\boldsymbol{F}$, No difference in peak amplitude of $C \mathrm{a}^{2+}$ transients in the open and closed arms. $\boldsymbol{G}, \mathrm{dmPFC}-\mathrm{BLA}$ mice show increased velocity of movement in the open arms than in the closed arms. $\boldsymbol{H}$, Once data are velocity corrected (see Materials and Methods), there is no longer a difference. $\boldsymbol{I}$, No difference in the frequency of calcium transients between the open arms and the closed arms (velocity corrected). $\boldsymbol{J}$, No difference in peak amplitude of calcium transients between the open arms and the closed arms (velocity corrected). $\boldsymbol{K}$, dmPFC-BLA projection neurons show no correlation between velocity and GCaMP signal. $\boldsymbol{L}$, Injection schematic with representative histology images showing targeting of DI0-GcaMP6m to the dmPFC and CAV-Cre/Syn-mCherry to the dorsomedial striatum. $\boldsymbol{M}$, Spatial analysis of frontostriatal projection activity in the EZM showing increased $\mathrm{Ca}^{2+}$ signal as they transition from the closed arms to the open arms (black line, transition point). The lowest and highest signals occur in the center of the closed and open arms, respectively. Individual animal data are sorted by the average signal across the closed arms (low to high). The average (average) heatmap is plotted below. $\boldsymbol{N}$, Perievent time histogram showing temporal decrease in $\mathrm{Ca}^{2+}$ signal on transition from open to closed arms. $\mathbf{0}, \mathrm{Ca}^{2+}$ signal is significantly lower in the closed arm post-transition (0-10 s) than in the open arm pretransition (-10 to 0 s). $\boldsymbol{P}$, Frequency of $\mathrm{Ca}^{2+}$ transients is significantly higher in the open arms than in the closed arms. $\mathbf{Q}$, Peak amplitude of $\mathrm{Ca}^{2+}$ transients is significantly higher in the open arms than in the closed arms. $\boldsymbol{R}, \mathrm{dmPFC}-\mathrm{DMS}$ mice show increased velocity of movement in the open arms compared with the closed arms. $\boldsymbol{S}$, Once data are velocity corrected (see Materials and Methods), there is no longer a difference. $\boldsymbol{T}$, No difference in frequency of calcium transients between the open arms and the closed arms (velocity corrected). $\boldsymbol{U}$, Peak amplitude of calcium transients is significantly higher in the open arms than in the closed arms (velocity corrected). $\boldsymbol{V}$, dmPFC-DMS projection neurons show no correlation between velocity and GCaMP signal. Error bars on all bar graphs represent SEM. ${ }^{*} p<0.033,{ }^{* *} p<0.002,{ }^{* * *} p<0.001$, ns, not significant.

projection-specific $\mathrm{Ca}^{2+}$ imaging through an implanted optical fiber $(400 \mu \mathrm{m})$ in the dmPFC.

Similar to previous analyses, we first plotted the spatial modulation of neural activity in each projection. In the dmPFC-BLA projection population, we found a mixture of responses: about half of the mice showed lower activity in the closed arms; and half showed no difference or the opposite trend. The findings were inconsistent across animals; the average spatial heatmap did not show a robust increased signal, as we observed with whole-population recording, as the mice moved further into the open arms (Fig. 3B). In the perievent time histogram, the trajectory of the dmPFC-BLA projection modulation also differed from the dmPFC whole-population data. Specifically, while the dmPFC whole-population data showed a marked increase in activity from baseline levels when in the open arm, the dmPFCBLA population showed no significant increase in the open arm, but rather a transient decrease in neural activity when the mice returned to the closed arms [Fig. 3C,D; two-way RM ANOVA interaction, $F_{(1,410)}=1.683, p=0.1953$; Sidak's multiple comparisons, $p=0.0112$ (GCaMP, pretransition vs post-transition); $p=0.3143$ (eYFP, pretransition vs post-transition); $N=164$ GCaMP transitions, $N=248$ eYFP transitions; $N=9$ GCaMP 
mice, $N=12$ eYFP mice]. Additionally, dmPFC-BLA projection neurons did not show any significant difference in the frequency of $\mathrm{Ca}^{2+}$ transients (Fig. $3 E$; paired $t$ test, $t=0.6235, \mathrm{df}=8$, $p=0.5503 ; N=9$ mice) or the amplitude of $\mathrm{Ca}^{2+}$ transient peaks (Fig. $3 F$; paired $t$ test, $t=0.1467, \mathrm{df}=8, p=0.8870 ; N=9$ mice) in open versus closed arms. Following velocity correction (Fig. $3 G, H$; prethreshold paired $t$ test, $t=4.034, \mathrm{df}=8, p=0.0038$; post-threshold paired $t$ test, $t=1.136, \mathrm{df}=8, p=0.2889 ; N=9$ mice), dmPFCBLA peak frequency and amplitude remained unchanged (Fig. $3 I, J$; frequency: paired $t$ test, $t=0.7882, \mathrm{df}=8, p=0.4533$; amplitude: paired $t$ test, $t=1.146, \mathrm{df}=9, p=0.2849$ ). There was no correlation between dmPFC-BLA neural signal and velocity (Fig. $3 K$; linear regression, signal $=-0.1918$ * (velocity) $\left.+0.6006, R^{2}=0.02,880\right)$.

Conversely, spatial and temporal activity of the dmPFC-DMS projection more closely resembled that of the dmPFC population as a whole (Fig. $3 M, N$ ), showing increased neural activity in the open arms, which decreased back to baseline levels following transition to the closed arm [Fig. 30; two-way RM ANOVA interaction, $F_{(1,653)}=6.039, p=0.0141$; Sidak's multiple comparisons, $p=0.0052$ (GCaMP, pretransition vs post-transition); $p=0.9937$ (eYFP, pretransition vs post-transition); $N=241$ GCaMP transitions, $N=414$ eYFP transitions; $N=10$ GCaMP mice, $N=10$ eYFP mice)]. Additionally, dmPFC-DMS projection neurons showed higher frequency (Fig. 3P; paired $t$ test, $t=2.408, \mathrm{df}=9, p=0.0393 ; N=10$ mice) and amplitude (Fig. 3Q; paired $t$ test, $t=3.504, \mathrm{df}=9, p=0.0067 ; N=10$ mice) of $\mathrm{Ca}^{2+}$ transients in the open arms than in the closed arms, similar to whole-population dmPFC recordings. Following velocity thresholding (Fig. 3R,S; prethreshold paired $t$ test, $t=4.829$, $\mathrm{df}=9, p=0.0009$; post-threshold paired $t$ test, $t=1.526$, $\mathrm{df}=9, p=0.1614 ; N=10$ mice), arm differences in peak frequency changed from significant $(p=0.0393)$ to not significant $(p=0.1060$; Fig. $3 T$; paired $t$ test, $t=1.796, \mathrm{df}=9$, $p=0.1060 ; N=10$ mice), but still showed a significantly higher peak amplitude in the open arms (Fig. $3 U$; paired $t$ test, $t=4.419, \mathrm{df}=9, p=0.0017 ; N=10$ mice). There was no correlation between dmPFC-BLA neural signal and velocity (Fig. $3 \mathrm{~V}$; linear regression, signal $=0.1474 *$ (velocity) $\left.1.024, R^{2}=0.08546\right)$. These data suggest that the dmPFCDMS population more robustly represents aspects of the innate avoidance task than the $\mathrm{dmPFC}-\mathrm{BLA}$ projection.

We confirmed that GCaMP and eYFP groups showed no significant difference in exploratory behavior on the EZM (Fig. $4 A$ : $\mathrm{dmPFC}$ whole population, unpaired $t$ test, $t=1.292, \mathrm{df}=18$, $p=0.2126, N=11$ GCaMP mice, $N=9$ eYFP mice; Fig. $4 B$ : dmPFC-BLA, unpaired $t$ test, $t=1.739, \mathrm{df}=21, \quad p=0.0967$, $N=11$ GCaMP, 12 eYFP; Fig. 4C: dmPFC-DMS, unpaired $t$ test, $t=1.777, \mathrm{df}=21, p=0.0900, N=13$ GCaMP, $10 \mathrm{eYFP}$ ) and verified correct placement of the fiber photometry optical fibers for all three photometry cohorts (Fig. $4 D-F$ ). Additionally, we performed histology to verify the specificity of our projection targeting by investigating whether dmPFC-BLA and dmPFC-DMS projection neurons had collateral projections to other brain regions. We found no detectable collaterals in the opposing downstream brain area (DMS for dmPFC-BLA projection, and BLA for dmPFC-DMS projection) as well as no visible collaterals in other areas of the brain (Fig. 4G-L). In accordance with previous studies (Gabbott et al., 2005; Little and Carter, 2013; Yizhar and Klavir, 2018), BLA-projecting dmPFC neurons were clustered in the more superficial cortical layers (Fig. 4G), while DMS-projecting neurons spanned across multiple cortical layers (Fig. 4J). To quantify the degree of infection for the two projections, we performed cell counts from the region below the fiber tip (Fig. $4 M, N$ ). Compared with the dmPFC-BLA group, there were significantly more cells labeled, with greater variation between animals, in the dmPFC-DMS group (Fig. 4O; unpaired $t$ test, $t=2.257, \mathrm{df}=14, p=0.0405, N=8 \mathrm{dmPFC}-\mathrm{DMS}$ slices, $N=8$ dmPFC-BLA slices, two slices per animal).

\section{Optogenetic stimulation of the dmPFC as a whole decreases avoidance, while inhibition has no effect}

Given our findings that endogenous activity of dmPFC-DMS projection neurons was highest in the open arms, we hypothesized that dmPFC inputs may provide a necessary source of excitation to drive exploratory behavior. As a first step, we tested the effect of non-projection-specific whole-population dmPFC pyramidal neuron optogenetic activation on exploratory behavior. We expressed CaMKII-ChR2-eYFP (ChR2) or CaMKII-eYFP (eYFP) and implanted an optical fiber $(200 \mu \mathrm{m})$ in the dmPFC to allow for in vivo optogenetic stimulation of dmPFC pyramidal cells during exploration of the EZM (Fig. 5A). We found that stimulating the dmPFC as a whole increased open arm exploration, decreasing avoidance behavior [Fig. 5B,C; two-way RM ANOVA interaction, $F_{(1,17)}=2.832, p=0.1107$; Sidak's multiple comparisons, $p=0.0360$ (ChR2, laser on vs off), $p=0.9845$ (eYFP, laser on vs off); $N=10$ ChR2 mice, $N=9$ eYFP mice] while having no effect on locomotion [Fig. 6D; two-way RM ANOVA interaction, $F_{(1,17)}=0.1599, p=0.6942$; Sidak's multiple comparisons, $p=0.2952$ (ChR2, laser on vs off), $p=0.6542$ (eYFP, laser on vs off); $N=10$ ChR2 mice, $N=9$ eYFP mice]. However, whole-population optogenetic inhibition using CaMKII-eNpHR3.0-eYFP (Fig. 5E) had no effect on open arm exploration [Fig. 5F,G; two-way RM ANOVA interaction, $F_{(1,18)}=1.833, p=0.1925$; Sidak's multiple comparisons, $p=0.6270(\mathrm{NpHR}$, laser on vs off); $p=0.5316$ (eYFP, laser on vs off); $N=10 \mathrm{NpHR}$ mice, $N=10$ eYFP mice] or locomotor activity [Fig. $5 H$; two-way RM ANOVA interaction, $F_{(1,18)}=0.3392, p=0.5675$; Sidak's multiple comparisons, $p=0.9699$ (NpHR, laser on vs off), $p=0.8022$ (eYFP, laser on vs off); $N=10 \mathrm{NpHR}$ mice, $N=10$ eYFP mice].

\section{Optogenetic manipulation of dmPFC-DMS projection neurons bidirectionally controls approach-avoidance behavior}

We then tested whether this effect on avoidance behavior was specifically mediated by the dmPFC-DMS projection. We used optogenetic manipulations to alter the activity of dmPFC-DMS projections, either augmenting (ChR2) or opposing (NpHR) the increase in activity naturally observed in the open arms in our fiber photometry recordings. To this end, we expressed ChR2 or eYFP in the dmPFC of mice and implanted an optical fiber in the DMS to stimulate dmPFC-DMS terminals during exploration of the EZM (Fig. 6A). ChR2 mice spent significantly more time exploring the open arms in laser-on epochs compared with laser-off epochs, and there was no effect of laser in eYFP animals [Fig. 6B-D; two-way RM ANOVA interaction, $F_{(1,14)}=14.92, p=0.0017$; Sidak's multiple comparisons, ${ }^{*} p<0.033,{ }^{* *} p<0.002,{ }^{* * *} p<0.001$ (ChR2, laser on vs off); $p=0.9256$ (eYFP, laser on vs off); $N=9$ ChR2 mice, $N=8$ eYFP mice]. Additionally, ChR2 mice spent significantly more time in the open arms during the last $5 \mathrm{~min}$ of the experiment (which includes the last 2 min of laser on) than during the prestimulation period (baseline, first $5 \mathrm{~min}$ ), while eYFP animals showed no difference [Fig. 6E; two-way RM ANOVA interaction, $F_{(1,15)}=14.44, p=0.0017$; Sidak's 
A

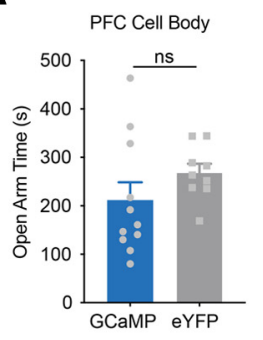

E

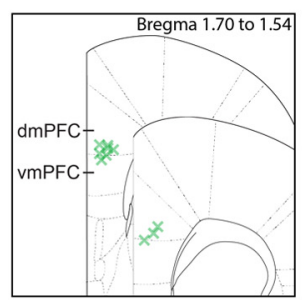

X GCaMP

G

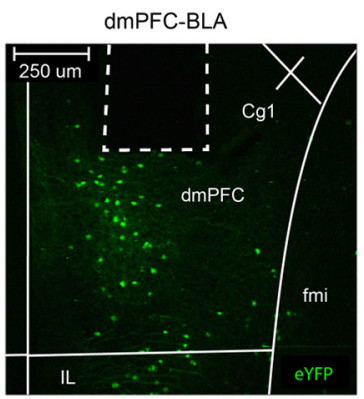

J

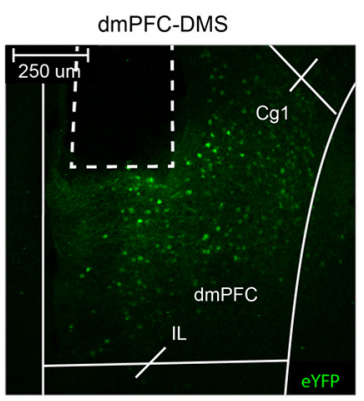

M

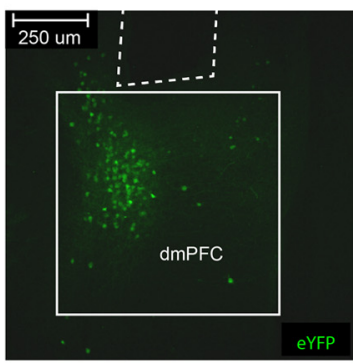

B
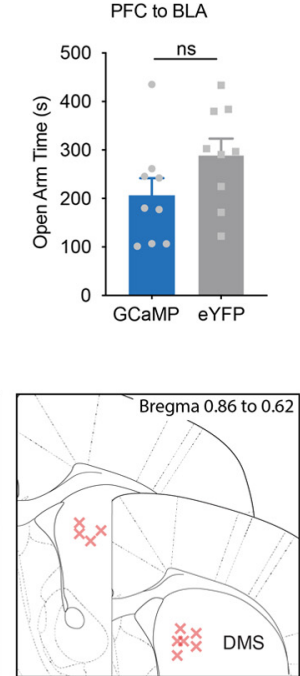

X Cav2-Cre + mChrerry

H

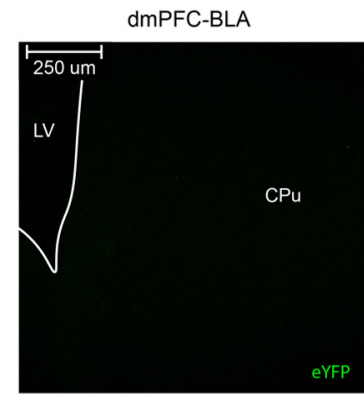

K

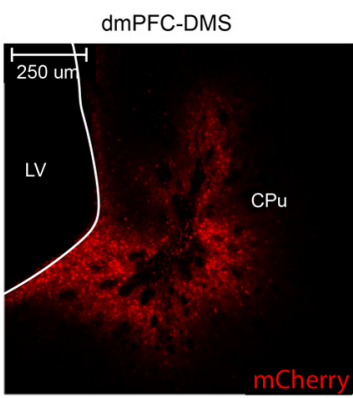

$\mathbf{N}$

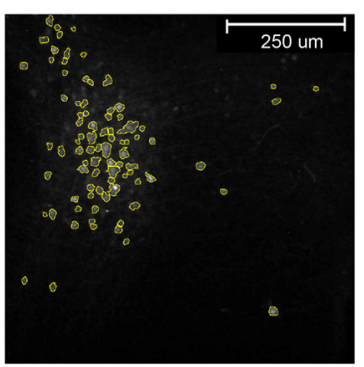

D

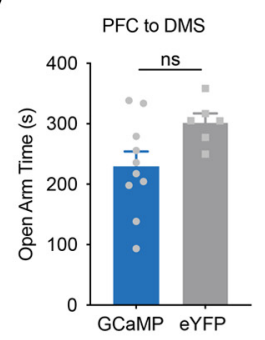

F
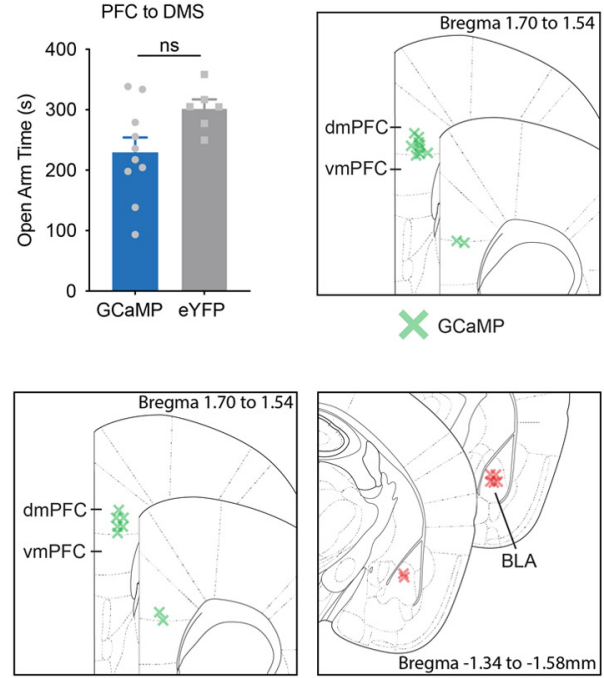

$X$ CAV2-Cre + mcherry

I

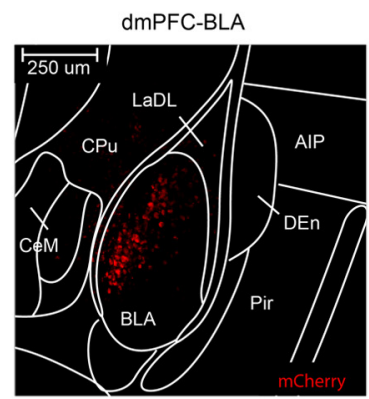

L

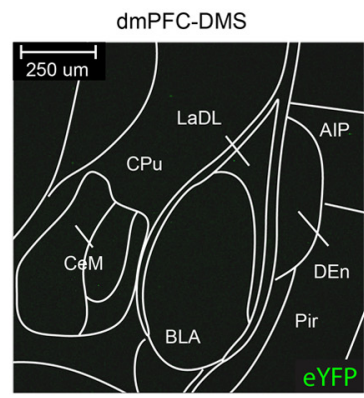

0

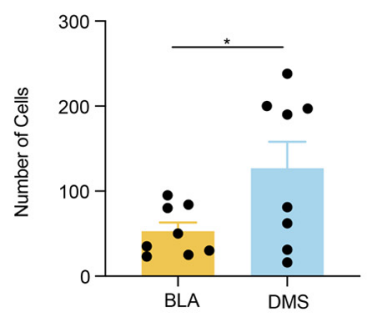

Figure 4. Behavioral and histologic analysis for photometry experiments. $A, \mathrm{dmPFC}$ whole-population mice show no difference in EZM exploratory behavior. $\boldsymbol{B}, \boldsymbol{C}$, Same as $\boldsymbol{A}$ for $\mathrm{dmPFC}-\mathrm{BLA}$ projection and dmPFC-DMS projection data, respectively. $\boldsymbol{D}$, dmPFC whole-population histologic verification of virus injection. $\boldsymbol{E}$, dmPFC-BLA histology. $\boldsymbol{F}$, dmPFC-DMS projection histology. Green crosses indicate targeting for GCaMP injections, and red crosses indicate targeting of CAV2-Cre injections. $G-L$, Histologic images showing no collaterals in opposing downstream brain region for the dmPFC-BLA projection population $(\mathbf{G}-\boldsymbol{I})$, as well as the dmPFC-DMS projection population $(\boldsymbol{J}-\mathbf{L})$. $\boldsymbol{M}$, Representative image of cell-counting slices. $\boldsymbol{N}, \mathbf{0}$, Quantification of the degree of virus infection for each of the projections. Error bars on all bar graphs represent SEM. ${ }^{*} p<0.033$, ns, not significant. 
A

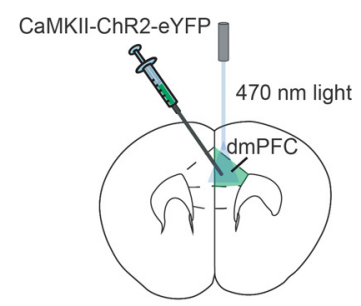

E

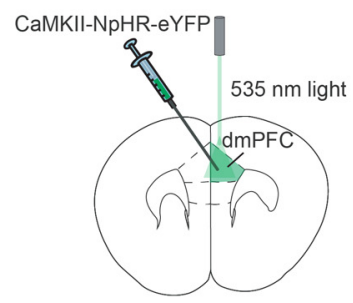

B

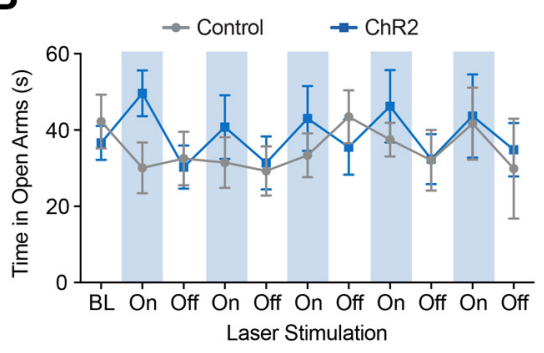

F

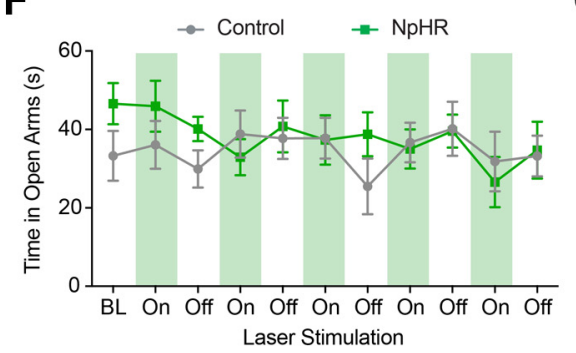

C

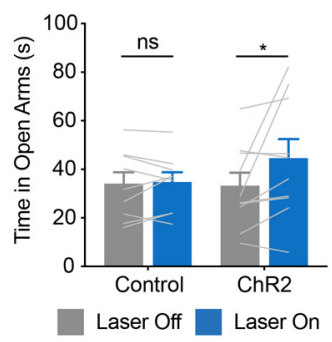

G

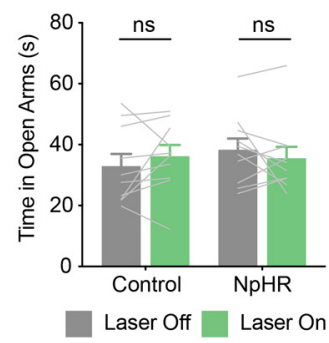

D

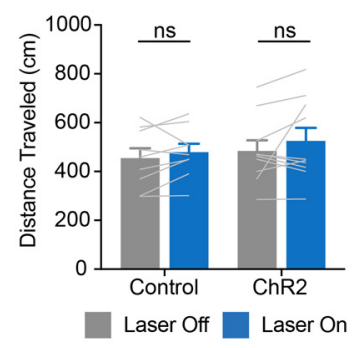

H

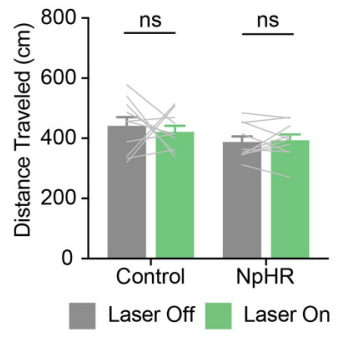

Figure 5. Optogenetic stimulation of the dmPFC as a whole decreases avoidance, while inhibition has no effect. $\boldsymbol{A}$, Schematic of optogenetic stimulation of dmPFC pyramidal neurons. CaMKII-ChR2-eYFP was virally expressed in the dmPFC, and a $200 \mu \mathrm{m}$ optical fiber was implanted above. Mice were optogenetically stimulated (470 nm light) during exploration of the EZM. $\boldsymbol{B}, \boldsymbol{C}$, ChR2 mice show increased time spent in the open arms during laser on epochs, while eYFP mice show no effect (5 min baseline followed by 2 min on/off epoch of laser stimulation). $\boldsymbol{D}$, Stimulation had no effect on locomotion for either group. $\boldsymbol{E}$, Schematic of optogenetic inhibition of dmPFC pyramidal neurons. CaMKII-NpHR-eYFP was virally expressed in the dmPFC, and a $200 \mu \mathrm{m}$ optical fiber was implanted above. Mice were optogenetically inhibited ( $535 \mathrm{~nm}$ light) during exploration of the EZM. $\boldsymbol{F}, \boldsymbol{G}$, NpHR and eYFP mice show no effect of laser stimulation on time spent in open arms during laser-on epochs (5 min baseline followed by $2 \mathrm{~min}$ on/off epoch of laser stimulation). $\boldsymbol{H}$, Inhibition had no effect on locomotion for either group. Error bars on all bar graphs represent SEM. ${ }^{*} p<0.033$, ns, not significant.

multiple comparisons, $p=0.0084$ (ChR2, first $5 \mathrm{~min}$ vs last $5 \mathrm{~min}$ ), $p=0.1142$ (eYFP, first $5 \mathrm{~min}$ vs last $5 \mathrm{~min}$ ); $N=9$ ChR2 mice, $N=8$ eYFP mice]. Laser stimulation had no effect on locomotion in either of the groups [Fig. 6F; RM two-way ANOVA interaction, $F_{(1,15)}=1.421, p=0.2517$; Sidak's multiple comparisons, $p=0.10852$ (ChR2, laser of vs laser on); $p=0.8889$ (eYFP, laser off vs laser on); $N=9$ ChR2 mice, $N=8$ eYFP mice].

While optogenetic stimulation of dmPFC-DMS terminals was sufficient to increase approach behavior in the EZM, we next tested whether activity in this pathway is necessary for normal approach-avoidance behavior. We expressed halorhodopsin in the dmPFC of mice and implanted an optical fiber in the downstream DMS to allow for optogenetic inhibition of projection terminals during exploration of the EZM (Fig. 7A). Optogenetic inhibition of these terminals in the NpHR group significantly decreased time spent in the open arms during the laser-on epochs relative to the laser-off epochs, with no effect on eYFP animals [Fig. $7 B, C$; two-way RM ANOVA interaction, $F_{(1,19)}=$ 1.911, $p=0.0 .1828$; Sidak's multiple comparisons, $p=0.0221$ (NpHR, laser on vs off); $p=0.7989$ (eYFP, laser on vs off); $N=12$ NpHr mice, $N=9$ eYFP mice]. NpHR mice also spent significantly less time in the open arms during the last 5 min compared with the first $5 \mathrm{~min}$ (prestimulation baseline), while eYFP animals showed no difference [Fig. 7D; two-way RM ANOVA interaction, $F_{(1,19)}=0.2739, p=0.6068$; Sidak's multiple comparisons, $p=0.0320(\mathrm{NpHR}$, first $5 \mathrm{~min}$ vs last $5 \mathrm{~min}$ ); $p=0.2386$ (eYFP, first $5 \mathrm{~min}$ vs last $5 \mathrm{~min}$ ); $N=12 \mathrm{NpHR}$ mice, $N=9$ eYFP mice]. Additionally, there was no effect of laser on locomotion within each of the NpHR and eYFP mice groups, although there was a significant overall effect of virus [Fig. 7E,F; RM two-way ANOVA interaction, $F_{(1,19)}=0.9647, p=0.3383$, virus effect $=0.0044,{ }^{*} p<0.05,{ }^{* *} p<0.004$; Sidak's multiple comparisons, $p=0.0 .9824$ (NpHR, laser of vs laser on); $p=0.3020$ (eYFP, laser off vs laser on); $N=12 \mathrm{NpHR}$ mice, $N=9$ eYFP mice]. These data suggest that activation of the dmPFC-DMS pathway is both necessary and sufficient for approach-avoidance behavior in the EZM.

\section{Optogenetic stimulation of dmPFC-DMS projection} terminals preferentially excites postsynaptic $D_{1}$ MSNs The results above indicate a role for the $\mathrm{dmPFC}-\mathrm{DMS}$ projection in approach-avoidance behavior, so we next investigated dmPFC-DMS connectivity. Using patch-clamp electrophysiology in striatal slices combined with terminal field optogenetic stimulation of dmPFC inputs, we assessed the responses of DMS $\mathrm{D}_{1}$ and $\mathrm{D}_{2} \mathrm{MSN}$ to excitation of dmPFC inputs. Sequential pairs of nearby $\mathrm{D}_{1}$ and $\mathrm{D}_{2}$ MSNs were patched in the whole-cell configuration (Fig. 8A), and both showed EPSCs in response to blue light stimulation (Fig. $8 B$ ). We plotted the ratio of EPSCs for each recorded pair $\left(D_{1}\right.$ and $D_{2}$; Fig. $\left.8 C\right)$; in almost all pairs, we observed larger EPSCs in $\mathrm{D}_{1}$ MSNs (Fig. $8 D$; Wilcoxon signedrank test, $p=0.0054 ; N_{\text {cell pairs }}=14$ ), yielding a ratio $>1$. These results indicate that the dmPFC projection preferentially activates $\mathrm{D}_{1} \mathrm{MSNs}$.

\section{Discussion}

We found that dmPFC pyramidal neurons on average exhibit an increase in activity during approach and exploration of the open arms of the EZM, corroborating previous studies showing that mPFC units distinguish between the open and closed arms (Adhikari et al., 2011). Given the increase in neural activity preceding entrance into the open arms, the dmPFC neurons may be responsive to the decision occurring at the transition point. In this "risk assessment" zone, an increase in neural activity would decrease avoidance of the open arms. The mPFC is well situated to play a critical role in processing innate avoidance behavior, as 
A

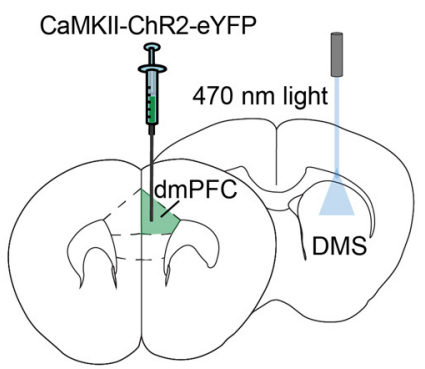

B

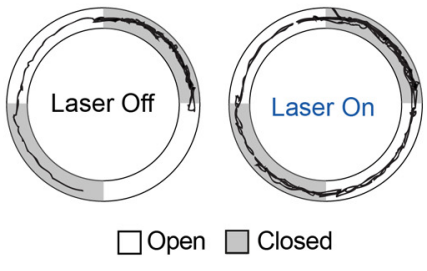

E

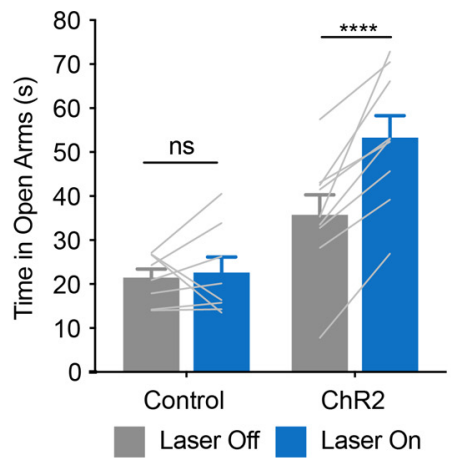

C

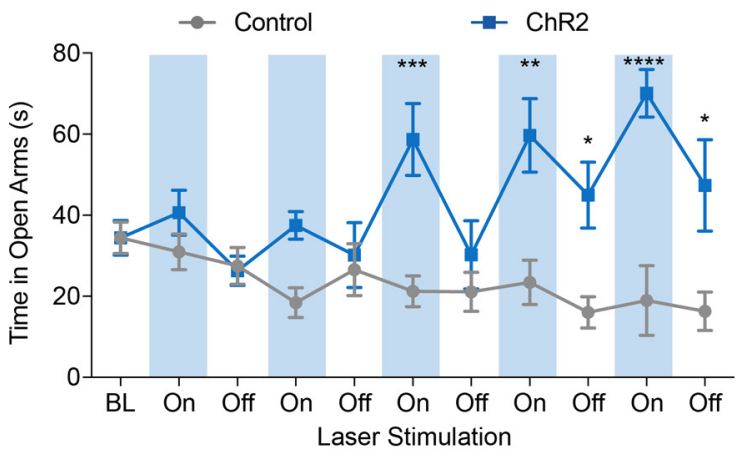

$\mathbf{F}$

Figure 6. Optogenetic stimulation of frontostriatal projection neurons decreases avoidance behavior. $\boldsymbol{A}$, Schematic showing optogenetic stimulation of dmPFC projections to the DMS. CaMKII-ChR2-eYFP was virally expressed in the dmPFC, and an optical fiber was implanted in the DMS to stimulate prefrontal terminals during exploration of the EZM. $\boldsymbol{B}$, Aerial view of representative individual animal path in the EZM showing increased exploration of the open arms during a single laser-on versus a single laser-off epoch. $\boldsymbol{C}$, ChR2 mice (blue line) show a selective increase in open arm exploration during laser-on epochs across the entire stimulation paradigm (consisting of alternating 2 min on/off epochs of laser stimulation). eYFP mice show no modulation of time spent in open arms in response to laser stimulation. $\boldsymbol{D}$, Quantification of the acute effects of laser stimulation on avoidance behavior. ChR2 mice show a significant increase in open arm time during laseron epochs compared with laser-off epochs. eYFP mice show no modulation of open arm time in laser-on versus laser-off epochs. $\boldsymbol{E}$, Quantification of lasting effects of laser stimulation on avoidance behavior. ChR2 but not eYFP mice show a significant increase in open arm time even beyond the laser stimulation period during the last 5 min in the EZM. $\boldsymbol{F}$, Both ChR2 and eYFP groups show no effect of laser stimulation on distance traveled. Error bars on all bar graphs represent SEM. ${ }^{*} p<0.033,{ }^{* *} p<0.002,{ }^{* * *} p<0.001,{ }^{* * * *} p<0.0001$, ns, not significant.

it receives inputs carrying contextual and valence information. Specifically, inputs from the BLA and ventral hippocampus to the $\mathrm{MPFC}$ are required for normal expression of innate avoidance behavior (Felix-Ortiz et al., 2016; Padilla-Coreano et al., 2016). However, little previous work has compared the roles of distinct efferent projections of the dmPFC in innate avoidance behavior. Here, we addressed this knowledge gap by investigating representation of innate avoidance behavior by frontostriatal and frontoamygdalar projection neurons. While $\mathrm{Ca}^{2+}$ signals from dmPFC-BLA projection neurons showed some modulation during open/closed arm transitions, there were no average changes in calcium peak amplitude and frequency between the open and closed arms, indicating no substantial or consistent differences in neural activity during the exploration of open versus closed arms of the EZM. This result was surprising given that many previous studies have focused on the dmPFC-BLA projection for its role in controlling fear expression (Sierra-Mercado et al., 2011; Tye et al., 2011; Courtin et al., 2014; Karalis et al., 2016) and have implicated the MPFC-BLA projection in safety signaling (Likhtik et al., 2014; Stujenske et al., 2014). In the context of these previous data, our results suggest that while the dmPFCBLA projection may be critically important for reflexive defensive behaviors such as freezing, a different top-down dmPFC projection may be more involved in the avoidance behaviors relevant to anxiety. This model is supported by a recent study in which optogenetic stimulation of the dmPFC-BLA projection did not affect innate avoidance behavior but did affect cued freezing during fear extinction retrieval (Adhikari et al., 2015).
We then turned to an alternative dmPFC projection target, the DMS, which is implicated in controlling action selection (Balleine et al., 2007), goal-directed actions (Hart et al., 2014), and, more recently, innate avoidance behavior (LeBlanc et al., 2018). The DMS is well situated to receive action initiation or inhibition signals from the dmPFC to facilitate avoidance behavior through its projection to downstream basal ganglia targets. Additionally, studies in previously stressed mice show the mPFC and DMS to be required for the development of stressor resistance (Amat et al., 2006, 2014; Strong et al., 2011). Of particular relevance is a recent study that investigated the role of the dmPFC-DMS projection in a learned approach-avoidance conflict task, which found that dmPFC-DMS projection neurons robustly increased activity during decision-making only under conflict conditions, but not during general value-based decisionmaking, suggesting that the dmPFC-DMS projection is particularly important for approach-avoidance conflict decision-making (Friedman et al., 2015). In alignment with this previous work, we found that dmPFC-DMS projection neurons robustly encoded aspects of innate avoidance in the EZM, with significantly greater activity in the open arms than in the closed arms, as well as spatial and temporal modulation of activity surrounding open/closed arm transitions, similar to what we observed with whole-population $\mathrm{dmPFC}$ recordings. These findings, combined with the previous work, suggest a model in which distinct subpopulations of dmPFC projection neurons play differential roles in anxiety-related behaviors, with the $\mathrm{dmPFC}-\mathrm{BLA}$ projection involved primarily in reflexive 
A

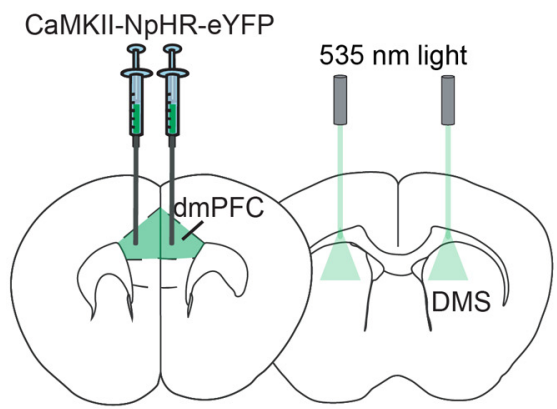

D

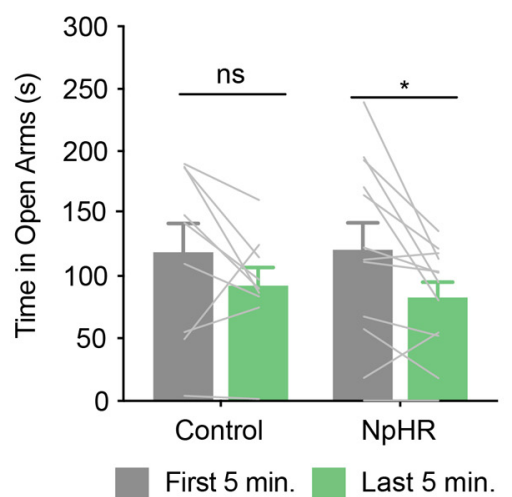

B

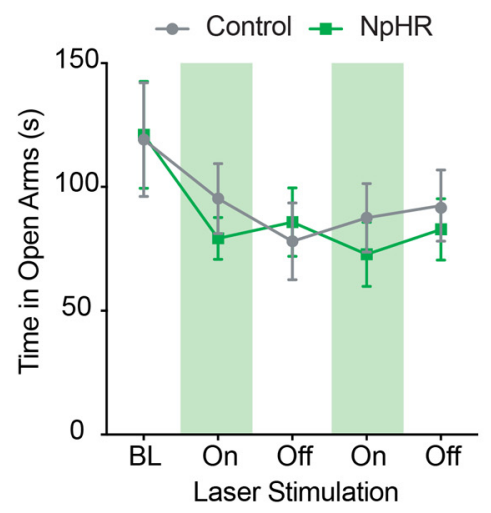

E

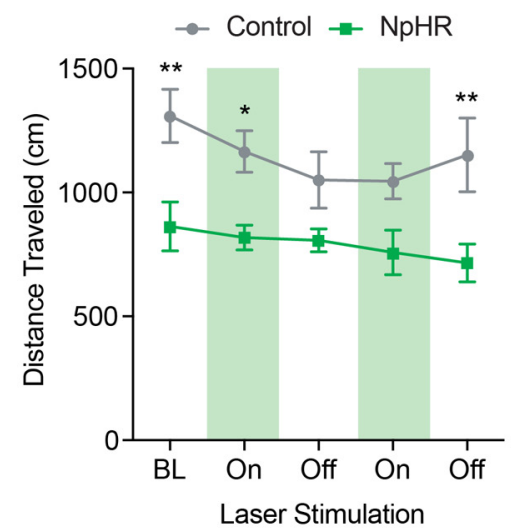

C

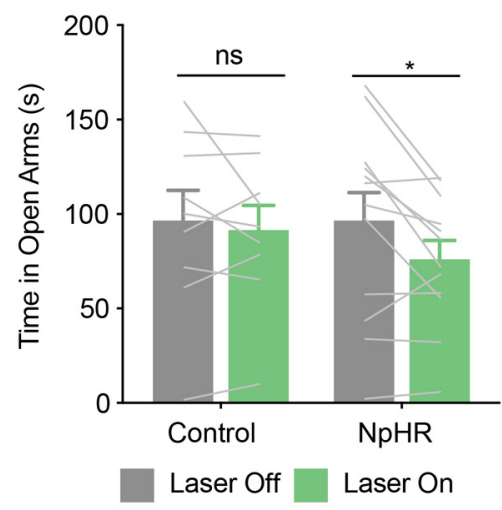

$\mathbf{F}$

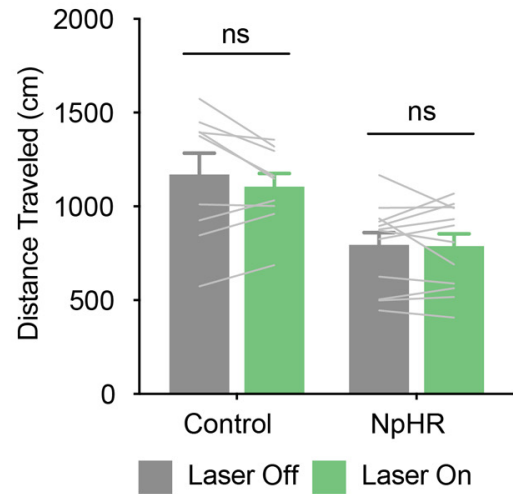

Figure 7. Optogenetic inhibition of frontostriatal projection neurons increases avoidance behavior. $\boldsymbol{A}$, Schematic of optogenetic inhibition of dmPFC-DMS neurons. CaMKII-NpHR-eYFP was virally expressed in the dmPFC, and a $200 \mu \mathrm{m}$ optical fiber was implanted in the DMS. Mice were optogenetically inhibited (535 nm light) during exploration of the EZM. B, C, NpHR mice show a decrease in time spent in the open arms during laser-on epochs compared with laser-off epochs. eYFP mice show no modulation of open arm time in laser-on versus laser-off epochs. $\boldsymbol{D}$, NpHR but not eYFP mice showed a significant decrease in open arm time between the first $5 \mathrm{~min}$ and the last $5 \mathrm{~min}$ in the EZM. $\boldsymbol{E}, \boldsymbol{F}$, While the NpHR and eYFP groups as a whole had different locomotor behavior, within each group NpHR and eYFP mice show no effect of laser stimulation on locomotion. Error bars on all bar graphs represent SEM. ${ }^{*} p<0.033,{ }^{* *} p<0.002$, ns, not significant.

A

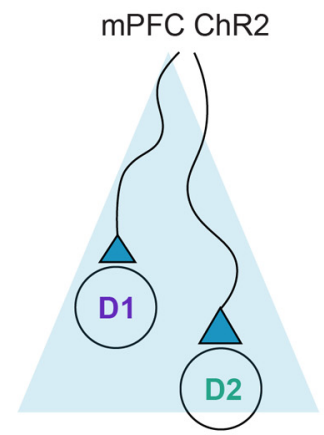

B

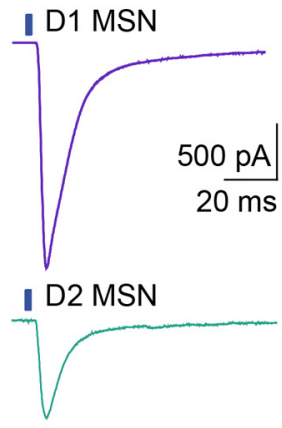

C

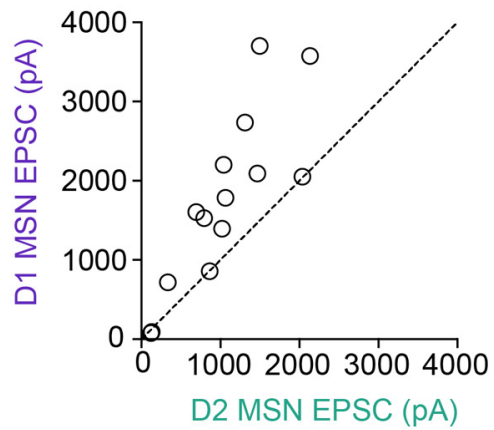

D

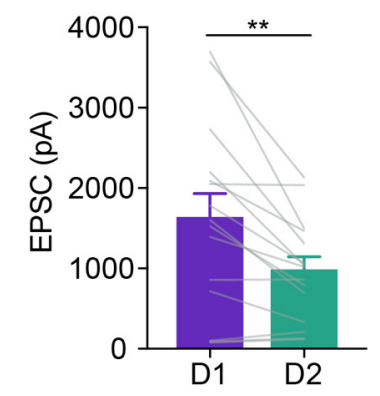

Figure 8. Optogenetic stimulation of dmPFC-DMS projection terminals preferentially excites postsynaptic $D_{1}$ MSNs. $A$, Schematic showing slice electrophysiology recording of striatal responses to optogenetic stimulation of dmPFC terminals. CaMKII-ChR2-eYFP was expressed in the dmPFC and recordings were taken in the DMS. $\boldsymbol{B}$, Representative traces from $D_{1}$ and $D_{2}$ MSNs in the DMS showing larger light-evoked EPSCs in $D_{1}$ MSNs. $C$, Sequential paired recordings from $D_{1}$ and $D_{2}$ MSNs (each circle represents one pair) showing stronger excitation of $D_{1}$ MSNs following stimulation of dmPFC-DMS projection terminals. $D, D_{1}$ MSNs show a significantly higher amplitude EPSC than $D_{2}$ MSNs in response to stimulation. Error bars on all bar graphs represent SEM. ${ }^{* *} p<0.002$.

fear behavior and the dmPFC-DMS projection more involved in anxious avoidance behavior.

Although our fiber photometry results show increased activity of dmPFC-DMS projection neurons during exploration of the open arms, it is not possible to interpret the directionality or valence of this signal from $\mathrm{Ca}^{2+}$ imaging alone. Theoretically, this increased signal could be interpreted in two opposing ways: as a correlate of increased "anxiety" that the animals experience after entering the open arms, or as a correlate of decreased anxiety that drove the animals into the open arms. To discriminate 
btween these two possibilities and causally link the dmPFCDMS projection to innate avoidance behavior, we used optogenetic manipulation in the EZM. We first found that global stimulation of the dmPFC moderately increased open arm exploration, while inhibition had no effect. When we moved to projection-specific optogenetic manipulation, we found that frontostriatal projection stimulation robustly increased open arm exploration, while inhibition decreased open arm exploration. These results, combined with our $\mathrm{Ca}^{2+}$ imaging data, suggest that the increase in endogenous frontostriatal activity in the open arms is likely a correlate of decreased avoidance or decreased anxiety-like behavior. This result is surprising given the classical role of the dmPFC in fear conditioning, in which increased dmPFC activity is associated with increased fear expression (Corcoran and Quirk, 2007; Burgos-Robles et al., 2009; Sotres-Bayon et al., 2012). Additionally, we observed a cumulative effect of repeated stimulation on the time spent in the open arms. Specifically, repeated laser stimulation of the dmPFC-DMS terminals led to an increase in open arm time in the later laser-off periods. This suggests that there may be plasticity occurring at dmPFC-DMS synapses that may be contributing to a lasting "anxiolytic" (decreased avoidance) effect. Future studies into the mechanisms of such plasticity in this circuit may be translationally beneficial in the development of therapeutic treatments to maintain long-lasting effects with minimal stimulation. When combined with our finding that global optogenetic stimulation of the dmPFC as a whole had a weaker effect on innate avoidance behavior, these results highlight the importance of considering projection specificity when addressing the heterogeneous dmPFC. Specifically, they suggest that there may be other dmPFC projection populations that promote avoidance when stimulated, canceling out the effects of dmPFC-DMS projection stimulation.

After identifying this novel role for dmPFC-DMS projections in encoding and controlling approach-avoidance behavior, we further characterized the dmPFC-DMS circuit at the synaptic level and investigated the role of different downstream cell types within the DMS. One previous rabies tracing study suggested that the dmPFC preferentially innervates striatal $D_{1}$ MSNs (Wall et al., 2013), while another study found similar innervation of $D_{1}$ and $\mathrm{D}_{2}$ MSNs (Guo et al., 2015). Using slice physiology, we confirmed that stimulation of dmPFC projection fibers in the DMS preferentially activated $\mathrm{D}_{1}$ MSNs, although we also found appreciable activation of $\mathrm{D}_{2}$ MSNs. These findings tie in well with a recent article that found stimulation of $\mathrm{D}_{2}$ MSNs to increase avoidance behavior (LeBlanc et al., 2018). Specifically, we propose that $D_{1}$ and $D_{2}$ MSNs may have opposing effects on avoidance behavior (similar to opposing roles in controlling locomotion and reward). We found that stimulation of dmPFCDMS projection terminals decreased avoidance behavior, and that stimulation of these terminals preferentially excites $D_{1}$ MSNs, suggesting that direct stimulation of $D_{1}$ MSNs in the DMS would also have an anxiolytic effect. This would balance nicely with the results from the study by LeBlanc et al. (2018) in creating an approach/avoidance balance system controlled by $\mathrm{D}_{1} / \mathrm{D}_{2}$ MSNs, respectively. Future experiments should examine the postsynaptic responses in the DMS to terminal stimulation of different input projection neurons as well as the intrastriatal mechanisms of $D_{1}$ and $D_{2}$ MSNs in controlling avoidance behavior.

Our data suggest a strong role of the dmPFC-DMS circuit in regulating avoidance behavior. Although our findings combined with previous work suggest that this circuit may more robustly regulate avoidance than the dmPFC-BLA circuit, there are several factors that could affect the direct comparison of neural signals between these two projection populations. First, we found that our retrograde viral targeting approach labeled a greater number of dmPFC-DMS cells than dmPFC-BLA cells. This difference in expression strength, combined with the known difference in cortical layer distribution of the two projection populations (Gabbott et al., 2005; Little and Carter, 2013; Yizhar and Klavir, 2018), could account for some differences in the magnitude of neural signals surrounding open/closed arm transitions, but would be unlikely to affect the shape of the PETH or the average changes in calcium transient frequency between open and closed arms. Last, while we showed differences in neural activity in the EZM and EPM tasks and theorized that these changes in activity represent approach-avoidance behavior, it is possible that these changes in neural signal may also be related to other features inherent in these tasks, such as risk-taking and physiological changes.

While previous studies have implicated the dmPFC and DMS separately in avoidance behavior, and have implicated the dmPFC-DMS circuit in decision-making under conflict (Friedman et al., 2015), our findings build on this previous work by providing direct evidence that dmPFC-DMS projection neurons are a novel population of dmPFC neurons involved in controlling anxiety-like behavior in the EZM, while the dmPFC-BLA pathway does not play a robust role. Our results support a model for prefrontal control of defensive behavior in which frontostriatal projection neurons modulate defensive actions such as avoidance, and frontoamygdalar projection neurons modulate defensive reactions such as freezing. This model may be solidified by further studies during fear behaviors to demonstrate selective recruitment of the dmPFC-BLA projection, not the dmPFC-DMS projection. Additionally, it is not known whether the role of dmPFC-DMS projection neurons is specific to innate avoidance behavior or more broadly involved in other types of learned avoidance behavior, such as active and passive avoidance. As such, future studies should compare the neural representations of these different types of avoidance behavior in a circuit-specific manner.

A core feature of human anxiety disorders is excessive avoidance behavior, which presents a barrier to treating these disorders. Our findings identify a novel frontostriatal projection population that controls innate avoidance behavior and may be a valuable target for future animal and human studies that seek to restore balance between approach and avoidance behaviors.

\section{References}

Adhikari A, Topiwala MA, Gordon JA (2011) Single units in the medial prefrontal cortex with anxiety-related firing patterns are preferentially influenced by ventral hippocampal activity. Neuron 71:898-910.

Adhikari A, Lerner TN, Finkelstein J, Pak S, Jennings JH, Davidson TJ, Ferenczi E, Gunaydin LA, Mirzabekov JJ, Ye L, Kim S-Y, Lei A, Deisseroth K (2015) Basomedial amygdala mediates top-down control of anxiety and fear. Nature 527:179-185.

Alexander GE, DeLong MR, Strick PL (1986) Parallel organization of functionally segregated circuits linking basal ganglia and cortex. Annu Rev Neurosci 9:357-381.

Amat J, Paul E, Zarza C, Watkins LR, Maier SF (2006) Previous experience with behavioral control over stress blocks the behavioral and dorsal raphe nucleus activating effects of later uncontrollable stress: role of the ventral medial prefrontal cortex. J Neurosci 26:13264-13272.

Amat J, Christianson JP, Aleksejev RM, Kim J, Richeson KR, Watkins LR, Maier SF (2014) Control over a stressor involves the posterior dorsal striatum and the act/outcome circuit. Eur J Neurosci 40:2352-2358. 
Aupperle RL, Martin PP (2010) Neural systems underlying approach and avoidance in anxiety disorders. Dialogues Clin Neurosci 12:517.

Aupperle RL, Melrose AJ, Francisco A, Paulus MP, Stein MB (2015) Neural substrates of approach-avoidance conflict decision-making. Hum Brain Mapp 36:449-462.

Balleine BW, Delgado MR, Hikosaka O (2007) The role of the dorsal striatum in reward and decision-making. J Neurosci 27:8161-8165.

Beck AT (1979) Cognitive therapy and the emotional disorders. New York: Penguin.

Bijttebier P, Beck I, Claes L, Vandereycken W (2009) Gray's reinforcement sensitivity theory as a framework for research on personality-psychopathology associations. Clin Psychol Rev 29:421-430.

Blanchard DC, Griebel G, Pobbe R, Blanchard RJ (2011) Risk assessment as an evolved threat detection and analysis process. Neurosci Biobehav Rev 35:991-998.

Bryant RA, Kemp AH, Felmingham KL, Liddell B, Olivieri G, Peduto A, Gordon E, Williams LM (2008) Enhanced amygdala and medial prefrontal activation during nonconscious processing of fear in posttraumatic stress disorder: an fMRI study. Hum Brain Mapp 29:517-523.

Burgos-Robles A, Vidal-Gonzalez I, Quirk GJ (2009) Sustained conditioned responses in prelimbic prefrontal neurons are correlated with fear expression and extinction failure. J Neurosci 29:8474-8482.

Cardinal RN, Parkinson JA, Hall J, Everitt BJ (2002) Emotion and motivation: the role of the amygdala, ventral striatum, and prefrontal cortex. Neurosci Biobehav Rev 26:321-352.

Carr DB, Sesack SR (1996) Hippocampal afferents to the rat prefrontal cortex: synaptic targets and relation to dopamine terminals. J Comp Neurol 369:1-15.

Christakou A, Robbins TW, Everitt BJ (2004) Prefrontal cortical-ventral striatal interactions involved in affective modulation of attentional performance: implications for corticostriatal circuit function. J Neurosci 24:773-780.

Corcoran KA, Quirk GJ (2007) Activity in prelimbic cortex is necessary for the expression of learned, but not innate, fears. J Neurosci 27:840-844.

Corr PJ (2002) J.A. Gray's reinforcement sensitivity theory: tests of the joint subsystems hypothesis of anxiety and impulsivity. Pers Individ Dif 33:511-532.

Corr PJ (2004) Reinforcement sensitivity theory and personality. Neurosci Biobehav Rev 28:317-332.

Courtin J, Chaudun F, Rozeske RR, Karalis N, Gonzalez-Campo C, Wurtz H, Abdi A, Baufreton J, Bienvenu TC, Herry C (2014) Prefrontal parvalbumin interneurons shape neuronal activity to drive fear expression. Nature 505:92.

Coutlee CG, Huettel SA (2012) The functional neuroanatomy of decision making: prefrontal control of thought and action. Brain Res 1428:3-12.

Domenech P, Koechlin E (2015) Executive control and decision-making in the prefrontal cortex. Curr Opin Behav Sci 1:101-106.

Do-Monte FH, Manzano-Nieves G, Quiñones-Laracuente K, Ramos-Medina L, Quirk GJ (2015) Revisiting the role of infralimbic cortex in fear extinction with optogenetics. J Neurosci 35:3607-3615.

Felix-Ortiz AC, Burgos-Robles A, Bhagat ND, Leppla CA, Tye KM (2016) Bidirectional modulation of anxiety-related and social behaviors by amygdala projections to the medial prefrontal cortex. Neuroscience 321:197-209.

Friedman A, Homma D, Gibb LG, Amemori K-i, Rubin SJ, Hood AS, Riad MH, Graybiel AM (2015) A corticostriatal path targeting striosomes controls decision-making under conflict. Cell 161:1320-1333.

Gabbott PL, Warner TA, Jays PR, Salway P, Busby SJ (2005) Prefrontal cortex in the rat: projections to subcortical autonomic, motor, and limbic centers. J Comp Neurol 492:145-177.

Gittis AH, Nelson AB, Thwin MT, Palop JJ, Kreitzer AC (2010) Distinct roles of GABAergic interneurons in the regulation of striatal output pathways. J Neurosci 30:2223-2234.

Gourley SL, Taylor JR (2016) Going and stopping: dichotomies in behavioral control by the prefrontal cortex. Nat Neurosci 19:656-664.

Grace AA, Floresco SB, Goto Y, Lodge DJ (2007) Regulation of firing of dopaminergic neurons and control of goal-directed behaviors. Trends Neurosci 30:220-227.

Graybiel AM, Aosaki T, Flaherty AW, Kimura M (1994) The basal ganglia and adaptive motor control. Science 265:1826-1831.
Green RH, Beatty WW, Schwartzbaum J (1967) Comparative effects of septo-hippocampal and caudate lesions on avoidance behavior in rats. J Comp Physiol Psychol 64:444.

Groenewegen HJ, Wright CI, Uylings HB (1997) The anatomical relationships of the prefrontal cortex with limbic structures and the basal ganglia. J Psychopharmacol 11:99-106.

Guo Q, Wang D, He X, Feng Q, Lin R, Xu F, Fu L, Luo M (2015) Wholebrain mapping of inputs to projection neurons and cholinergic interneurons in the dorsal striatum. PLoS One 10:e0123381.

Hart G, Leung BK, Balleine BW (2014) Dorsal and ventral streams: the distinct role of striatal subregions in the acquisition and performance of goal-directed actions. Neurobiol Learn Mem 108:104-118.

Hoover WB, Vertes RP (2007) Anatomical analysis of afferent projections to the medial prefrontal cortex in the rat. Brain Struct Funct 212:149-179.

Hosokawa T, Kennerley SW, Sloan J, Wallis JD (2013) Single-neuron mechanisms underlying cost-benefit analysis in frontal cortex. J Neurosci 33:17385-17397.

Karalis N, Dejean C, Chaudun F, Khoder S, Rozeske RR, Wurtz H, Bagur S, Benchenane K, Sirota A, Courtin J (2016) 4- Hz oscillations synchronize prefrontal-amygdala circuits during fear behavior. Nat Neurosci 19:605.

Kim CK, Ye L, Jennings JH, Pichamoorthy N, Tang DD, Yoo AW, Ramakrishnan C, Deisseroth K (2017) Molecular and circuit-dynamical identification of top-down neural mechanisms for restraint of reward seeking. Cell 170:1013-1027.e14.

Kravitz AV, Kreitzer AC (2012) Striatal mechanisms underlying movement, reinforcement, and punishment. Physiology 27:167-177.

Kravitz AV, Tye LD, Kreitzer AC (2012) Distinct roles for direct and indirect pathway striatal neurons in reinforcement. Nat Neurosci 15:816.

LeBlanc KH, London TD, Szczot I, Bocarsly ME, Friend DM, Nguyen KP, Mengesha MM, Rubinstein M, Alvarez VA, Kravitz AV (2018) Striatopallidal neurons control avoidance behavior in exploratory tasks. Mol Psychiatry 25:491- 415.

Lerner TN, Shilyansky C, Davidson TJ, Evans KE, Beier KT, Zalocusky KA, Crow AK, Malenka RC, Luo L, Tomer R (2015) Intact-brain analyses reveal distinct information carried by SNc dopamine subcircuits. Cell 162:635-647.

Likhtik E, Stujenske JM, Topiwala MA, Harris AZ, Gordon JA (2014) Prefrontal entrainment of amygdala activity signals safety in learned fear and innate anxiety. Nat Neurosci 17:106-113.

Little JP, Carter AG (2013) Synaptic mechanisms underlying strong reciprocal connectivity between the medial prefrontal cortex and basolateral amygdala. J Neurosci 33:15333-15342.

McDonald A (1991) Organization of amygdaloid projections to the prefrontal cortex and associated striatum in the rat. Neuroscience 44:1-14.

Padilla-Coreano N, Bolkan SS, Pierce GM, Blackman DR, Hardin WD, Garcia-Garcia AL, Spellman TJ, Gordon JA (2016) Direct ventral hippocampal-prefrontal input is required for anxiety-related neural activity and behavior. Neuron 89:857-866.

Peters YM, O'Donnell P, Carelli RM (2005) Prefrontal cortical cell firing during maintenance, extinction, and reinstatement of goal-directed behavior for natural reward. Synapse 56:74-83.

Pinto L, Dan Y (2015) Cell-type-specific activity in prefrontal cortex during goal-directed behavior. Neuron 87:437-450.

Qiu C, Liao W, Ding J, Feng Y, Zhu C, Nie X, Zhang W, Chen H, Gong Q (2011) Regional homogeneity changes in social anxiety disorder: a resting-state fMRI study. Psychiatry Res 194:47-53.

Rothman AH, Glick SD (1976) Differential effects of unilateral and bilateral caudate lesions on side preference and passive avoidance behavior in rats. Brain Res 118:361-369.

Schott BH, Minuzzi L, Krebs RM, Elmenhorst D, Lang M, Winz OH, Seidenbecher CI, Coenen HH, Heinze H-J, Zilles K, Duzel E, Bauer A (2008) Mesolimbic functional magnetic resonance imaging activations during reward anticipation correlate with reward-related ventral striatal dopamine release. J Neurosci 28:14311-14319.

Sesack SR, Grace AA (2010) Cortico-basal ganglia reward network: microcircuitry. Neuropsychopharmacology 35:27.

Sesack SR, Deutch AY, Roth RH, Bunney BS (1989) Topographical organization of the efferent projections of the medial prefrontal cortex in the rat: an anterograde tract-tracing study with Phaseolus vulgaris leucoagglutinin. J Comp Neurol 290:213-242. 
Shafiei N, Gray M, Viau V, Floresco SB (2012) Acute stress induces selective alterations in cost/benefit decision-making. Neuropsychopharmacology 37:2194

Shuen JA, Chen M, Gloss B, Calakos N (2008) Drd1a-tdTomato BAC transgenic mice for simultaneous visualization of medium spiny neurons in the direct and indirect pathways of the basal ganglia. J Neurosci 28:26812685.

Sierra-Mercado D, Padilla-Coreano N, Quirk GJ (2011) Dissociable roles of prelimbic and infralimbic cortices, ventral hippocampus, and basolateral amygdala in the expression and extinction of conditioned fear. Neuropsychopharmacology 36:529-538.

Sotres-Bayon F, Quirk GJ (2010) Prefrontal control of fear: more than just extinction. Curr Opin Neurobiol 20:231-235.

Sotres-Bayon F, Sierra-Mercado D, Pardilla-Delgado E, Quirk GJ (2012) Gating of fear in prelimbic cortex by hippocampal and amygdala inputs. Neuron 76:804-812.

Strong PV, Christianson JP, Loughridge AB, Amat J, Maier SF, Fleshner M, Greenwood BN (2011) 5-hydroxytryptamine 2C receptors in the dorsal striatum mediate stress-induced interference with negatively reinforced instrumental escape behavior. Neuroscience 197:132144.

Stujenske JM, Likhtik E, Topiwala MA, Gordon JA (2014) Fear and safety engage competing patterns of theta-gamma coupling in the basolateral amygdala. Neuron 83:919-933.

Tye KM, Prakash R, Kim S-Y, Fenno LE, Grosenick L, Zarabi H, Thompson KR, Gradinaru V, Ramakrishnan C, Deisseroth K (2011) Amygdala circuitry mediating reversible and bidirectional control of anxiety. Nature 471:358.

Wall NR, De La Parra M, Callaway EM, Kreitzer AC (2013) Differential innervation of direct-and indirect-pathway striatal projection neurons. Neuron 79:347-360.

Wiesendanger E, Clarke S, Kraftsik R, Tardif E (2004) Topography of cortico-striatal connections in man: anatomical evidence for parallel organization. Eur J Neurosci 20:1915-1922.

Yizhar O, Klavir O (2018) Reciprocal amygdala-prefrontal interactions in learning. Curr Opin Neurobiol 52:149-155.

Zhao X-H, Wang P-J, Li C-B, Hu Z-H, Xi Q, Wu W-Y, Tang X-W (2007) Altered default mode network activity in patient with anxiety disorders: an fMRI study. Eur J Radiol 63:373-378. 